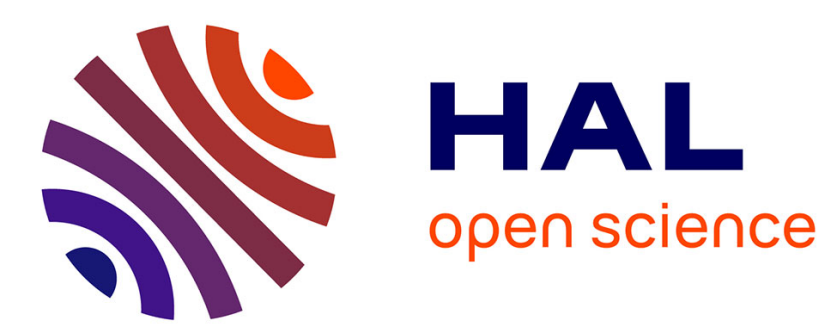

\title{
Fast and efficient shear-force assisted production of covalently functionalized oxide nanosheets
}

Frédéric Payet, Corinne Bouillet, Fabrice Leroux, Cédric Leuvrey, Pierre

Rabu, François Schosseler, Christine Taviot-Guého, Guillaume Rogez

\section{To cite this version:}

Frédéric Payet, Corinne Bouillet, Fabrice Leroux, Cédric Leuvrey, Pierre Rabu, et al.. Fast and efficient shear-force assisted production of covalently functionalized oxide nanosheets. Journal of Colloid and Interface Science, 2022, 10.1016/j.jcis.2021.08.213 . hal-03336183

\section{HAL Id: hal-03336183 \\ https://hal.science/hal-03336183}

Submitted on 7 Sep 2021

HAL is a multi-disciplinary open access archive for the deposit and dissemination of scientific research documents, whether they are published or not. The documents may come from teaching and research institutions in France or abroad, or from public or private research centers.
L'archive ouverte pluridisciplinaire HAL, est destinée au dépôt et à la diffusion de documents scientifiques de niveau recherche, publiés ou non, émanant des établissements d'enseignement et de recherche français ou étrangers, des laboratoires publics ou privés. 


\title{
Fast and efficient shear-force assisted production of covalently functionalized oxide nanosheets
}

Frédéric Payet, ${ }^{a}$ Corinne Bouillet, ${ }^{\mathrm{a}}$ Fabrice Leroux, ${ }^{\mathrm{b}}$ Cédric Leuvrey, ${ }^{\mathrm{a}}$ Pierre Rabu, ${ }^{\mathrm{a}}$ François Schosseler, ${ }^{\mathrm{c}}$ Christine Taviot-Guého ${ }^{\mathrm{b}}$ and Guillaume Rogez ${ }^{\mathrm{a}}$

a Institut de Physique et Chimie des Matériaux de Strasbourg, CNRS - Université de Strasbourg, UMR7504, 23 rue du Loess, BP43, 67034 Strasbourg cedex 2, France (frederic.payet@ipcms.unistra.fr; corinne.bouillet@ipcms.unistra.fr; leuvrey@ipcms.unistra.fr; pierre.rabu@ipcms.unistra.fr; rogez@unistra.fr)

${ }^{\mathrm{b}}$ Institut de Chimie de Clermont-Ferrand, Université Clermont Auvergne, UMR CNRS 6296, Clermont Auvergne INP, 24 av Blaise Pascal, BP 80026, 63171 Aubière cedex, France (fabrice.leroux@uca.fr; christine.taviot-gueho@uca.fr) 'Institut Charles Sadron, CNRS UPR 22, 23 rue du Loess, BP84047, 67034 Strasbourg cedex 2, France (chossel@unistra.fr)

\begin{abstract}
Hypothesis

While controlled and efficient exfoliation of layered oxides often remains a time consuming challenge, the surface modification of inorganic nanosheets is of outmost importance for future applications. The functionalization of the bulk material prior to exfoliation should allow the application of tools developped for Van der Waals materials to directly produce functionalized oxide nanosheets.

Experiments

The Aurivillius phase $\mathrm{Bi}_{2} \mathrm{SrTa}_{2} \mathrm{O}_{9}$ is functionalized by a linear aliphatic phosphonic acid via microwave-assisted reactions. The structure of the hybrid material and the coordination of the phosphonate group is scrutinized, notably by Pair Distribution Function. This functionalized layered oxide is then exfoliated in one hour in organic solvent, using high shear force dispersion. The obtained nanosheets are characterized in suspension and as deposits to check their chemical integrity.

Findings

The covalent functionalization decreases the electrostatic cohesion between the inorganic layers leading to an efficient exfoliation in short time under shearing. The functionalization of the bulk material is preserved on the nanosheets upon exfoliation and plays a major role to enable liquid-phase exfoliation and in the stability of the resulting suspensions. This strategy is very promising for the straighforward preparation of functionalized nanosheets, paving the way for versatile design of new (multi)functional hybrid nanosheets for various potential applications.
\end{abstract}

\section{Keywords}

Layered perovskite, microwave-assisted functionalization, Pair Distribution Function, shear-force exfoliation, functionalized nanosheets, light scattering 


\section{Introduction}

Inorganic nanosheets have attracted attention for long, and generate a renewed and ever growing interest since the discovery of the outstanding properties of graphene.[1] Such systems can find applications in various domains : nanofillers in polymer-based materials, [2] energy and environmental applications,[3-5] or magnetic materials in spintronics[6,7] to quote just a few.[8-10] Nanosheets can also be used in nanoarchitectonics to design multifunctional "artificial solids" from their controlled reassembly[11-15] or heterostructures from their interstratification, so-called intercalative hybridization.[16]

Nanosheets are usually obtained by exfoliation of layered materials. For instance, the micromechanical cleavage, "scotch and tape", of Van der Waals materials, graphite or metal dichalcogenides, has enabled to study the physical properties of individual nanosheets. $[1,17]$ Yet this process may be well adapted for academic purposes even if it is not shape-controlled, but is definitely not industrially scalable and cannot be applied to a broad range of layered materials. Indeed, liquid-phase exfoliation methods are thus more commonly developed and used, either assisted by surfactant, cation or polymer insertion, or by shear-forces or sonication.[18-22]

Among all interesting materials, the exfoliation of ion-exchangeable layered transition metal oxides, and more particularly those with a perovskite-like structure, is particularly investigated as they offer a large versatility in terms of chemical composition or layer thickness and can provide a large panel of properties (ferroelectricity, luminescence, photovoltaics or catalytic properties for instance).[23,24] For these compounds composed of charged layers, the classical exfoliation method consists in inserting a bulky organo-ammonium ion (typically tetrabutylammonium) in between the layers. This leads to a swelling phenomenon in water which substantially increases the interlamellar space until the total separation of the inorganic slabs. [20]

Yet, besides the exfoliation itself, the surface modification of the inorganic nanosheets is of outmost importance, since it allows to process the nanosheets suspensions in organic solvents, as well as offers a possible tool to control their lateral size, size distribution and properties.[25] At this stage, two strategies are debated: one consisting in introducing organic functionalization prior to exfoliation, the other consisting in exfoliating first, using classical methods, and then to attach functional groups onto the separated nanosheets.

Despite the difficulty to functionalize oxide nanosheets after their exfoliation while avoiding their reaggregation,[26] the latter approach has led to the development of efficient procedures, either by direct functionalization,[27] or using microwave-assisted reactions,[28,29] or reactions in a biphasic system,[30] recently complemented by the use of microchannels to improve the yields.[31]

The former approach, functionalization prior to exfoliation, was initially limited to Dion-Jacobson niobiate phases. [32,33] Recently this strategy has been further explored and developed in the case of layered titanates by the group of $Y$. Oaki.[25,26,34-37] They demonstrated that functionalization of layered titanates by amines followed by swelling and subsequent exfoliation in organic non-polar solvents allows not only to obtain functionalized nanosheets but also to control the lateral sizes and tune the properties such as bandgap energies. Up to now, this investigation has been essentially limited to layered Ti, $\mathrm{W}$ and $\mathrm{Mn}$ oxides functionalized by amines.[38] Therefore, the question of its application to other materials and functionalization is still largely open, particularly with a covalent link between the inorganic slabs and the organic molecule.

Actually one of the great advantages of layered perovskites is that they can be easily functionalized, as bulk layered materials, by various molecules, amines, alcohols or carboxylic or phosphonic acids.[23] This time consuming functionalization step has long been considered as a major drawback of the functionalization prior to exfoliation strategy to 
obtain decorated nanosheets. Nevertheless, the development of microwave assisted reactions has recently considerably sped up the functionalization processes, [39-43] thus enabling to overcome this difficulty.

In this article, we describe the microwave-assisted preparation and characterization of a new hybrid layered perovskite derived from the Aurivillius phase $\mathrm{Bi}_{2} \mathrm{SrTa}_{2} \mathrm{O}_{9}$ (BST) and its subsequent exfoliation. The bulk hybrid material functionalized by decylphosphonic acid is exfoliated using high-shear force disperser, a process usually used to exfoliate Van der Waals materials, leading to stable colloidal suspensions of covalently functionalized nanosheets. The obtained nanosheets are characterized as deposits and in colloidal suspension, revealing a mean lateral size of $c a .800 \mathrm{~nm}$, and a thickness comprised between one to six single-layers.

\section{Materials and methods}

All the reactants and solvents were used without further purification. The parent bulk materials $\mathrm{Bi}_{2} \mathrm{SrTa}_{2} \mathrm{O}_{9}$ (BST), $\mathrm{H}_{2} \mathrm{Bi}_{0.1} \mathrm{Sr}_{0.85} \mathrm{Ta}_{2} \mathrm{O}_{7}$ (HST) and $\left(\mathrm{CH}_{3}\left(\mathrm{CH}_{2}\right)_{3} \mathrm{NH}_{3}\right)_{0.8} \mathrm{H}_{1.2} \mathrm{Bi}_{0.1} \mathrm{Sr}_{0.85} \mathrm{Ta}_{2} \mathrm{O}_{7} .3 \mathrm{H}_{2} \mathrm{O}$ ( $\mathbf{C}_{4} \mathbf{N}$-HST) were prepared according to published procedures. [41,44-46]

Microwave syntheses were performed with a Monowave 300 (Anton Paar) synthesis reactor in non-acidic conditions and with a MultiwaveGO (Anton Paar) reactor when aqueous acidic conditions were required.

Caution: even though no problem was encountered in the course of this research, special care must be taken while working with sealed glass containers. Containers must be carefully checked for any defect, and replaced if necessary to avoid possible explosion. Concentrated hydrochloric acid, used to prepare HST, is corrosive and irritating.

Synthesis of $\left(\mathrm{C}_{10} \mathrm{H}_{21} \mathrm{PO}_{2}(\mathrm{OH})\right)_{0.9} \mathrm{H}_{1.1} \mathrm{Bi}_{0.1} \mathrm{Sr}_{0.85} \mathrm{Ta}_{2} \mathrm{O}_{7} .2 \mathrm{H}_{2} \mathrm{O}\left(\mathrm{C}_{10} \mathrm{PO}_{3}-\mathrm{HST}\right): 100 \mathrm{mg}(0.14 \mathrm{mmol})$ of $\mathrm{C}_{4} \mathrm{~N}$-HST are dispersed in 9 $\mathrm{mL}$ of THF and $266 \mathrm{mg}(1.2 \mathrm{mmol})$ of 1-decylphosphonic acid. The mixture was sealed in a $30 \mathrm{~mL}$ vial and heated by microwave irradiation at $110{ }^{\circ} \mathrm{C}$ for $180 \mathrm{~min}$ (maximum incident power: $70 \mathrm{~W}$ ). In these conditions, the autogenous pressure was about 4 bars. The obtained white powder was washed with acetone by three centrifugations, each of them performed at $14000 \mathrm{rpm}$ (18400 g) for $5 \mathrm{~min}$, then air-dried. Anal. Calcd for $\left(\mathrm{C}_{10} \mathrm{H}_{21} \mathrm{PO}_{2}(\mathrm{OH})\right)_{0.9} \mathrm{H}_{1.1} \mathrm{Bi}_{0.1} \mathrm{Sr}_{0.85} \mathrm{Ta}_{2} \mathrm{O}_{7} .2 \mathrm{H}_{2} \mathrm{O}(\mathrm{M}=791.1 \mathrm{~g} / \mathrm{mol}): \mathrm{H}$, 2.73; C, 13.41; P, 3.46; Ta, 44.92. Found: H, 2.74; C, 13.79; P, 3.3; Ta, 46.00\%. Elemental analysis also showed the presence of $\mathrm{N}(0.41 \%)$ attributed to traces of remaining butylamine from $\mathbf{C}_{4} \mathbf{N}-\mathrm{HST}$.

Elemental analyses: $\mathrm{C}, \mathrm{N}$ and $\mathrm{H}$ elemental analyses were carried out at the Service Commun d'Analyses of the University of Strasbourg. The Ta and $\mathrm{P}$ contents of $\mathbf{C}_{10} \mathbf{P O}_{3}-\mathbf{H S T}$ and of the nanosheets dispersion were determined at the Plateforme d'Analyse of the School of Chemistry Polymers and Materials of Strasbourg by inductively coupled plasma-atomic emission spectroscopy (ICP-AES), after dissolution in a mixture of hydrochloric, nitric and fluoric acids at $170^{\circ} \mathrm{C}$ in a closed autoclave.

Powder X-ray diffraction (PXRD) patterns were collected in a Bragg-Brentano geometry with a Bruker D8ADVANCE diffractometer (CuK $\alpha 1=0.1540598 \mathrm{~nm}$ ) equipped with a LynxEye detector discriminating in energy.

The atomic Pair Distribution Functions were obtained from X-ray total scattering data collected on a PANalytical Empyrean diffractometer equipped with a solid state GaliPIX3D detector, a focusing X-ray multilayer mirror, and an Ag anticathode $\left(K \alpha_{1}=0.5594214 \AA\right.$ 员 $\alpha_{2}=0.5638120 \AA$ ). Powder samples were mounted in glass capillaries of $0.7 \mathrm{~mm}$ diameter. Data were recorded over the range $1<2 \theta<145^{\circ}$, which corresponds to an accessible maximum value for the scattering vector $Q$ max of $21.4 \AA^{-1}$. An optimized variable counting time strategy has been adopted to counteract the decrease of the scattered intensity at the highest angles because of the X-ray form factor. An empty capillary of the same type was measured in the same way for background subtraction. The total time of the measurement was $20 \mathrm{~h}$, the same for both the sample and 
the empty capillary. Data merging, background subtraction, and $\mathrm{K} \alpha_{2}$ stripping were done using HighScore Plus software provided by PANalytical Corporation. The program PDFgetX3 was used to generate corrected and normalized total scattering structure functions $S(Q)$.[47] The PDF or $G(r)$ were calculated from the Fourier transforms of the reduced structure functions truncated at $21 \AA^{-1}$. Finally, the program PDFgui was used to extract local structural information from the measured PDF. [48]

FT-IR spectra were collected in ATR mode on a Spectrumll spectrometer (Perkin-Elmer).

Solid state ${ }^{13} \mathrm{C}$ and ${ }^{31} \mathrm{P}$ NMR spectra were recorded with a 300 Bruker spectrometer at 75.47 or $121.49 \mathrm{MHz}$ respectively, using magic angle spinning (MAS) condition at $10 \mathrm{kHz}$ and a $4 \mathrm{~mm}$ diameter size zirconia rotor. ${ }^{13} \mathrm{C}$ spectra obtained by proton enhanced cross-polarization method (CP) were referenced to the carbonyl of the glycine calibrated at 176.03 ppm. Recycling and Hartman-Hahn contact times were $5 \mathrm{~s}$ and $1250 \mu \mathrm{s}$, respectively. Spinal $64{ }^{1} \mathrm{H}$ phase-decoupling was applied during ${ }^{13} \mathrm{C}$ channel acquisition. ${ }^{31} \mathrm{P}$ spectra were calibrated to $\mathrm{H}_{3} \mathrm{PO}_{4}$ at $0.0 \mathrm{ppm}$, and recorded after a $\pi / 2$ simple impulsion.

Shear-force assisted liquid exfoliation: exfoliations were carried out using a high-shear force disperser (T10 basic UltraTurrax from IKA) holding a S10N-10G shaft (rotor-stator gap of $0.2 \mathrm{~mm})$. Typically, $20 \mathrm{mg}\left(2.5 \cdot 10^{-2} \mathrm{mmol}\right)$ of layered materials were dispersed in $40 \mathrm{~mL}$ of isopropanol at a shear-mixer speed of $11000 \mathrm{rpm}$ for 6 cycles of 10 min dispersion followed by 20 min pause (total dispersion time of $60 \mathrm{~min}$ ). The resulting dispersion passed through a selective centrifugation process: starting at $400 \mathrm{rpm}$ (15 g) and up to $1600 \mathrm{rpm}$ (135 g) in $400 \mathrm{rpm}$ steps (5 min each). At each step the bottom is removed and the supernatant is kept for the next centrifugation. Finally, the exfoliated material was collected by centrifugation at 14000 rpm (18400 g) for $20 \mathrm{~min}$ and redispersed in $20 \mathrm{~mL}$ of isopropanol giving rise to a stable suspension.

Transmission Electron Microscopy (TEM) studies were conducted on suspensions deposited onto a lacey-carbon-coated $\mathrm{Cu}$ grid. Special care must be paid to remove all traces of residual solvent before insertion of the grid in the microscope to prevent contamination of the sample under the electron beam due to solvent degradation. Therefore, the as-prepared grid was stored under vacuum overnight before its insertion into the microscope. The images were obtained with a JEOL $2100 \mathrm{~F}$, corrected from the spherical aberration on the condenser lens, allowing to reach $0.18 \mathrm{~nm}$ resolution at $100 \mathrm{kV}$ in STEM mode. This high voltage was chosen in order to reduce knock-on damage, visible when observed at $200 \mathrm{kV}$.[49]

Scanning Electron microscopy (SEM) images were obtained using a JEOL 6700F microscope operating at 25 kV in STEM mode (T-SEM). Sample preparation is identical to the one used for TEM.

Atomic Force Microscopy (AFM) was performed with a Digital Instrument 3100 microscope. The nanomaterials were deposited on a $\mathrm{Si} / \mathrm{SiO}_{2}$ substrate which was cleaned (ultrasonication during $30 \mathrm{~min}$ in isopropanol followed by $\mathrm{O}_{2}$ plasma cleaning) and pre-coated with polyethyleneimine (PEI) (dip-coating in a solution of $2 \mathrm{mg} \cdot \mathrm{mL}^{-1}$ of PEI at pH =9). Typically, the nanosheet suspension was drop-casted onto the pre-coated substrate and the solvent was left to evaporate. The images were recorded in tapping mode, using the ScanAsyst ${ }^{\circledR}$ optimization (Bruker).

Light scattering experiments were performed using a compact ALV/CGS-8 goniometer equipped with a He-Ne laser (14 $\mathrm{mW}, \lambda=632.8 \mathrm{~nm}$, vertically polarized) and an ALV-7002/USB-25 correlator. The temperature of the matching index fluid (toluene) was kept at $25 \pm 0.1^{\circ} \mathrm{C}$ using a circulating water bath. The scattered light was repeatedly recorded for 1 min at different detector positions i.e. at different scattering vectors $q$ defined as:

$$
q=\frac{4 \pi n}{\lambda} \sin \frac{\theta}{2}
$$

where $\mathrm{n}$ is the solvent refractive index, $\lambda$ the laser wavelength and $\theta$ the angle between the detector position and the direction of the incident beam. As the scattering intensity was found to evolve in time, measurements were cycled for several 
hours to characterize the kinetics of this evolution. All the scattering intensities were normalized by the intensity scattered by a toluene standard measured at $90^{\circ}$ angle and $25^{\circ} \mathrm{C}$.

The autocorrelation functions of the scattered electromagnetic field were analyzed with the CONTIN software[50,51] using a set of scripts described elsewhere.[52] These scripts allowed a batch analysis of the large number of measurements generated by the experimental procedure.

\section{Results and discussion}

\subsection{Synthesis and characterization of the bulk layered hybrid material $\mathrm{C}_{10} \mathrm{PO}_{3}-\mathrm{HST}$}

The synthesis of the title compound derives from previous works on the microwave-assisted functionalization of Ta-oxide and $\mathrm{Nb}$-oxide with perovskite structure by amines or alcohols, by us[41-43] and others.[39,40] Even though a preintercalation step remains necessary, the use of solvothermal and microwave conditions allows to speed up considerably the reaction times with respect to classical reaction conditions ( $3 \mathrm{~h}$ vs. $48 \mathrm{~h}$ ).[53]

Fig. 1 shows the PXRD patterns of HST, $\mathbf{C}_{4} \mathbf{N}-\mathbf{H S T}$ and $\mathbf{C}_{10} \mathbf{P O}_{3}$-HST. The layered structure of the compounds is evidenced by the intense low angle series of peaks corresponding to $(00 /)$ reflections. As expected, the interlayer distance increases with the size of the inserted molecule: $10.2 \AA$ for HST, $20.6 \AA$ for $\mathbf{C}_{4} \mathbf{N}$-HST and $32.6 \AA$ for $\mathbf{C}_{10} \mathbf{P O}_{3}$-HST. The out-of-plane reflections assigned to (100), (110) and (200) planes (at $22.68^{\circ}, 32.59^{\circ}$ and $46.32^{\circ}$ respectively (Cu K $\left.\alpha 1\right)$ ) in the XRD pattern of HST are also found in the PXRD patterns of the hybrid materials. It suggests the inorganic perovskite-like structure of the slabs remains unchanged after microwave-assisted functionalization which is thus a topotactic reaction.

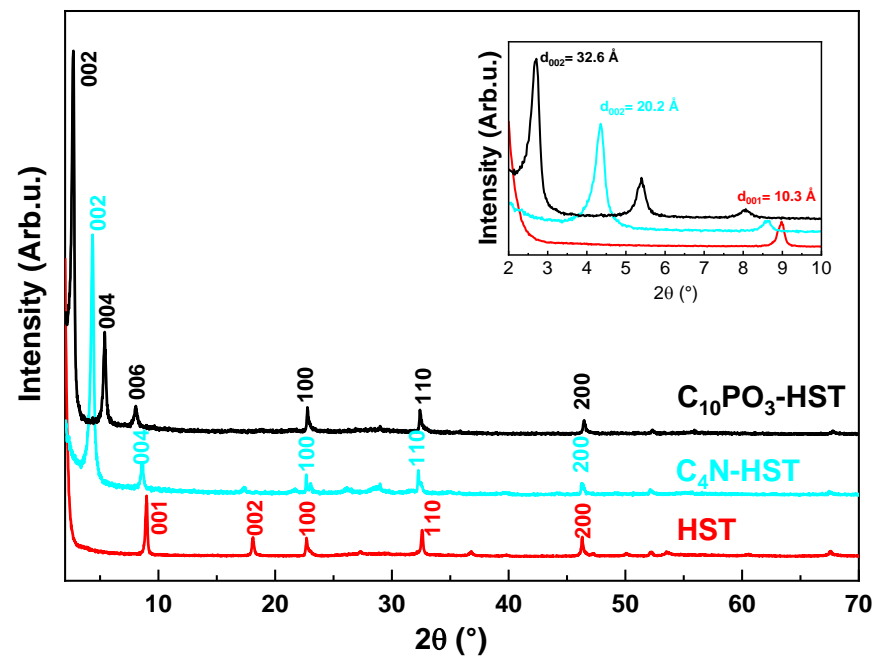

Fig. 1 XRD patterns of HST (red), $\mathrm{C}_{4} \mathbf{N}-\mathrm{HST}$ (cyan) and $\mathrm{C}_{10} \mathrm{PO}_{3}-\mathrm{HST}$ (black).

The mean crystalline domain size in $\mathbf{C}_{10} \mathbf{P O}_{3}$-HST powder was estimated using the Scherrer formula, to $40 \mathrm{~nm}$ in the $c$ direction and to $60 \mathrm{~nm}$ in the $a b$-direction. These values are similar to the ones obtained for HST and amine-functionalized derivatives.[41] It is worth underlining that this coherently scattering domain size corresponds to the mean size of defectfree crystallites. The lateral size of the inorganic slabs can be estimated from SEM images to be around $2 \mu \mathrm{m}$ whereas their thickness is around $1 \mu \mathrm{m}$ (Fig. S1).

The infrared spectra of all compounds are shown in Fig. 2 and confirm the functionalization of the layered materials. The most intense feature in all spectra is visible at $580 \mathrm{~cm}^{-1}$ and corresponds to the Ta-O elongation vibration. The intensity of the 
bands between 2800 and $3000 \mathrm{~cm}^{-1}$, corresponding to $\mathrm{C}-\mathrm{H}$ vibrations, increases as the carbon chains length increases. As for $\mathrm{C}_{10} \mathrm{PO}_{3}$-HST, its spectrum shows the characteristic $\mathrm{P}-\mathrm{C}$ stretching vibration band at $1465 \mathrm{~cm}^{-1}$ which is also present in the spectrum of 1-decylphosphonic acid. It evidences that the integrity of the molecule is maintained after microwave reaction. Meanwhile the bands in the 1350-400 $\mathrm{cm}^{-1}$ range, corresponding to the phosphonic acid part, are different in the layered hybrid with respect to the 1-decylphosphonic acid molecule alone. The P-O stretching band $\left(1013 \mathrm{~cm}^{-1}\right)$ is broadened whereas the intensity of the $\mathrm{P}-\mathrm{OH}$ stretching band $\left(920 \mathrm{~cm}^{-1}\right)$ is lowered in $\mathbf{C}_{10} \mathbf{P O}_{3}-\mathbf{H S T}$. In addition, the $\mathrm{P}=\mathrm{O}$ stretching band (around $1200 \mathrm{~cm}^{-1}$ ) is only hardly distinguishable in the hybrid compound. These observations are in line with what has been described for the grafting of phosphonic acids onto titanium oxide particles or into Dion-Jacobson phase niobium oxide and confirm a covalent grafting of the 1-decylphosphonic acid onto the inorganic layers. $[53,54]$

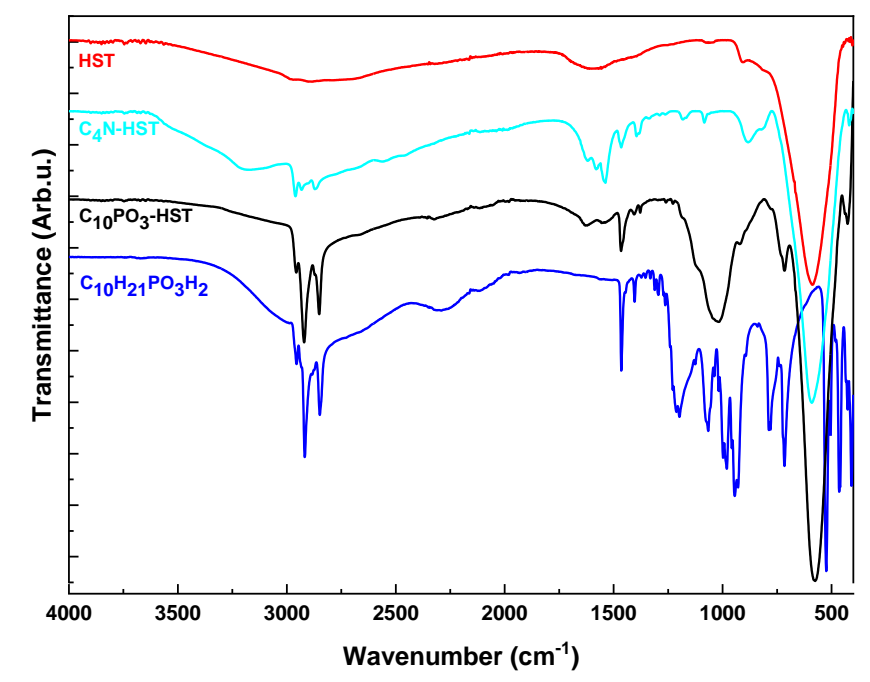

Fig. 2 Infrared spectra of HST (red), $\mathrm{C}_{4} \mathrm{~N}-\mathrm{HST}$ (cyan), $\mathrm{C}_{10} \mathrm{PO}_{3}$-HST (black)

and 1-decylphosphonic acid (blue)

Solid-state ${ }^{13} \mathrm{C}$ CP/MAS NMR spectrum of $\mathbf{C}_{10} \mathbf{P O}_{3}$-HST confirms the successful insertion of 1-decylphosphonic acid (Fig. 3). The assignment was performed using ChemNMR in the Chemdraw ${ }^{\circledR}$ suite. In addition, the NMR spectrum evidences the presence of an impurity at $20.4 \mathrm{ppm}$, attributed to traces of butylamine (in keeping with elemental analysis showing $0.41 \%$ of N). It has not been possible to remove this impurity. Since no trace of $\mathbf{C}_{4} \mathbf{N}-\mathbf{H S T}$ was observed in the PXRD pattern of $\mathbf{C}_{10} \mathbf{P O}_{3}{ }^{-}$ HST (see Fig. 1), the impurity has been ascribed to incomplete exchange during the reaction from $\mathbf{C}_{4} \mathbf{N}-\mathbf{H S T}$ to $\mathbf{C}_{10}$ PO ${ }_{3}-\mathrm{HST}_{\text {. }}$ 


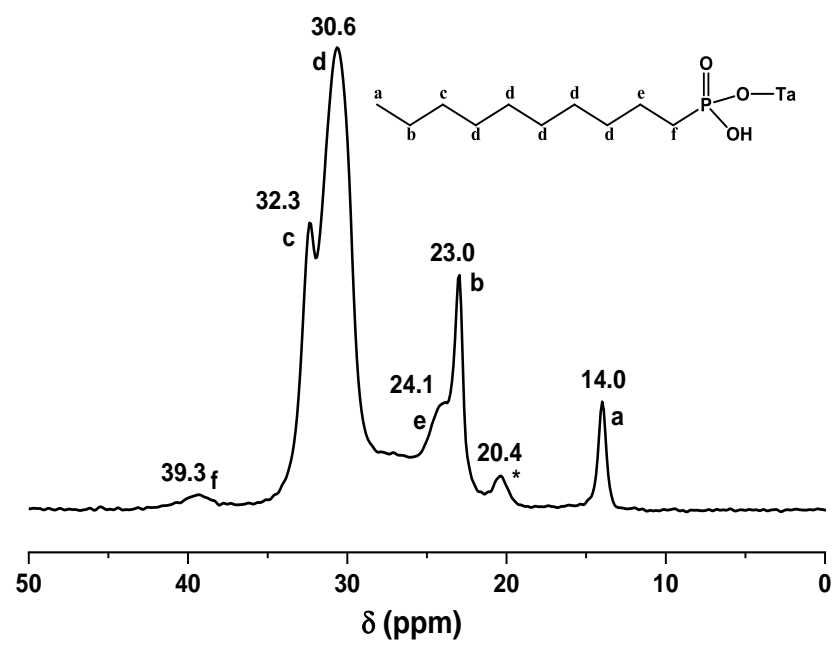

Fig. $3{ }^{13} \mathrm{CCP} / \mathrm{MAS}$ NMR spectrum of $\mathrm{C}_{10} \mathrm{PO}_{3}$-HST (the asterisk is attributed to the presence of an impurity of butylamine)

The solid-state ${ }^{31} \mathrm{P}$ MAS NMR spectrum of $\mathbf{C}_{10} \mathbf{P O}_{3}$-HST (Fig. S2) shows a broad signal at around 28 ppm, upfield-shifted by about $10 \mathrm{ppm}$ with respect to the 1-decylphosphonic acid spectrum, which confirms the grafting of the organic moiety to the tantalum oxide sheets.

In order to precise further the structure of the bulk material, total X-ray scattering and Pair Distribution Function analysis were carried out. This approach is especially powerful to get an accurate description of the short range structure of compounds exhibiting small crystalline domain size and high preferential orientation. The experimental PDF for $\mathbf{C}_{10} \mathbf{P O}_{3}-\mathrm{HST}$ sample is presented in Fig. 4. It shows a series of well-defined peaks at low $r$ and a rapid decay in the amplitude that may be attributed to a limited structural coherence $\sim 100 \AA$ in the stacking direction of the layers. Weak oscillations are still visible up to $200 \AA$.
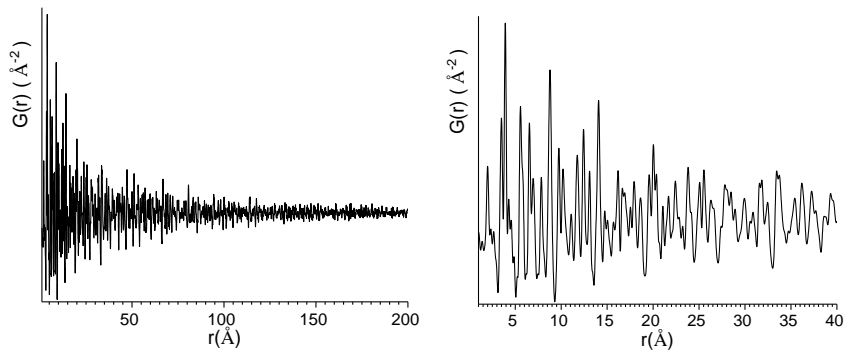

Fig. 4 (left) Experimental PDF for $\mathrm{C}_{10} \mathrm{PO}_{3}$-HST sample obtained up to $200 \AA$ and (right) details in the range from 1 to $40 \AA$

As a starting model, we considered the structural data reported by Whittle et al. for $\mathrm{Bi}_{2} \mathrm{SrTa}_{2} \mathrm{O}_{9}$ (BST) and the calculated PDF is displayed in Fig. 5a.[55] In this simulation, only the $\mathrm{SrTa}_{2} \mathrm{O}_{7}{ }^{2-}$ perovskite block was considered with a partial substitution of $\mathrm{Sr}$ by $\mathrm{Bi}$ in agreement with the experimental chemical composition of $\mathbf{C}_{\mathbf{1 0}} \mathbf{P O}_{\mathbf{3}}$-HST sample. To take into account the fact that the interlayer distance is $32.6 \AA$ in $\mathbf{C}_{10} \mathbf{P O}_{3}$-HST sample, the $c$ parameter of the orthorhombic cell was increased from $24.97 \AA$ to $65.2 \AA$ and the $z$ coordinates of the atoms the perovskite block were adjusted accordingly. The five main peaks labelled from $\mathrm{P} 1$ to $\mathrm{P} 5$ in the simulated PDF of the perovskite block are dominated by the contributions of M-O and M$\mathrm{M}$ pairs (with $\mathrm{M}=\mathrm{Ta}, \mathrm{Sr} / \mathrm{Bi}$ ) corresponding to the successive coordination shells around $\mathrm{M}$ cations as illustrated in Fig. $5 \mathrm{c}$. As expected, the experimental PDF of $\mathbf{C}_{10} \mathbf{P O}_{3}$-HST shares many common structural features with this model. However, distinct 
differences are also visible in particular at low $r$ due to the additional presence of the intercalated decylphosphonic $\mathrm{C}_{10} \mathrm{PO}_{3}$ chain.

The experimental PDF of 1-decylphosphonic acid used for the synthesis is also given in Fig. 5a. It shows two well defined peaks at $\sim 1.57 \AA$ including $\mathrm{P}-\mathrm{C} / \mathrm{P}-\mathrm{O} / \mathrm{C}-\mathrm{C}$ bonds and at $\sim 2.58 \AA$ corresponding to $\mathrm{C}---\mathrm{C}$ distance between second nearest neighbours as illustrated in Fig. 5b. Since these molecules are held together by Van der Waals interactions, an overlap of sharp and broad peaks is observed for $r$ above $\sim 3 \AA$ which is characteristic of the occurrence of both intramolecular and intermolecular distances. We can readily draw parallels with hybrid materials for which the variety of bonding interactions leads to very different widths of the PDF peaks and this is what makes the interpretation of PDF data of hybrid materials complicated as already reported elsewhere.[56,57] Handling the correlated motions to model the PDF data for molecular solids and hybrid materials over a wide range of $r$ is a current challenge.
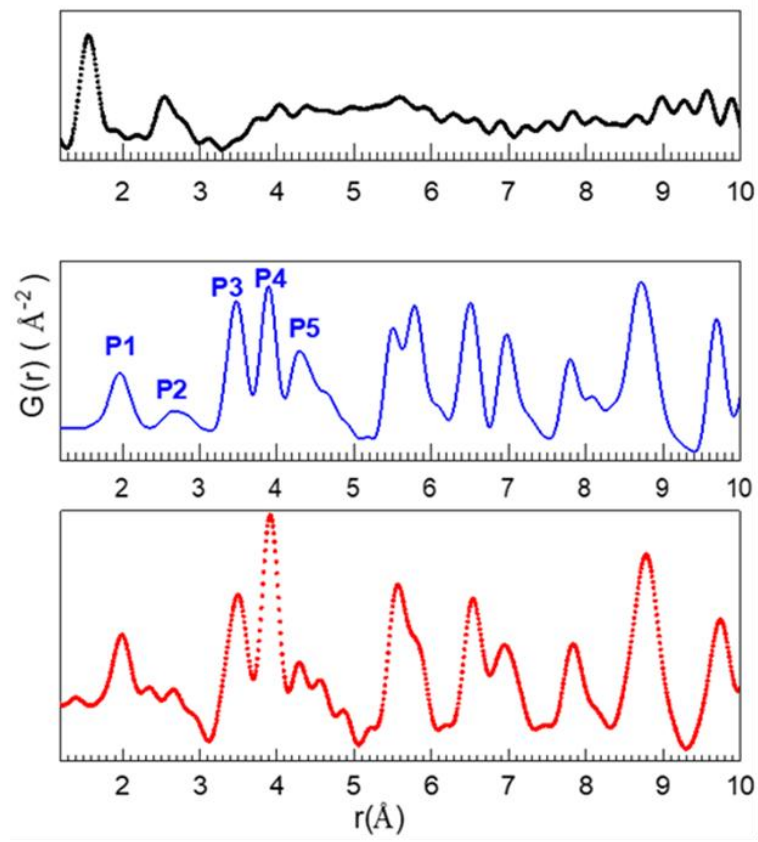

(a)

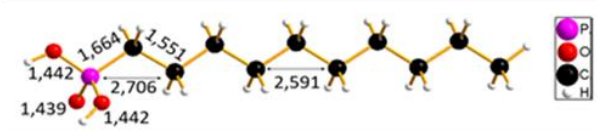

(b)

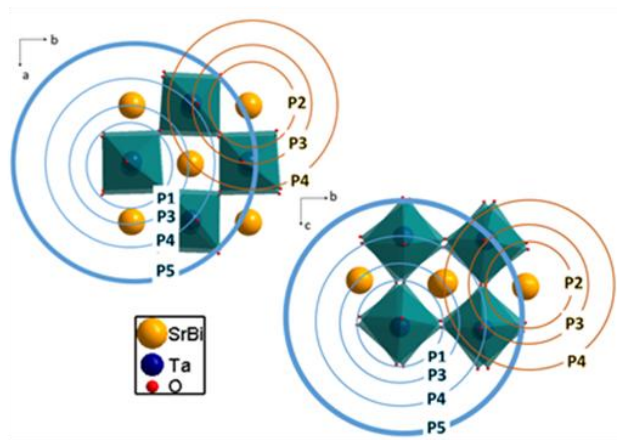

(c)

Fig. 5 (a) Experimental PDF for $\mathbf{C}_{10} \mathrm{PO}_{3}$-HST (bottom) compared to the model PDF for " $\mathrm{Sr}_{0.85} \mathrm{Bi}_{0.1} \mathrm{Ta}_{2} \mathrm{O}_{7}$ " perovskite layers (middle) and the experimental PDF for 1-decylphosphonic acid (top). Distances between nearest atoms (b) along the decylphosphonic chain and (c) in $\mathrm{Sr}_{0.85} \mathrm{Bi}_{0.1} \mathrm{Ta}_{2} \mathrm{O}_{7}$ perovskite layer model

In the case of the $\mathrm{C}_{10} \mathrm{PO}_{3}$-HST sample, we limited our scope to PDF data in the low $r$ region, where the distances refer only to near neighbour atoms, strongly bonded and dependent to each other, thus allowing the application of the common $r$ dependent peak broadening method.[58] As emphasized before, the first peaks in the PDF correspond to first atomic neighbour distances within the perovskite block and along intercalated decylphosphonic $\mathrm{C}_{10} \mathrm{PO}_{3}$, and also contain information about the nature of the bonding interaction between these two components. To extract quantitative information about the interaction between phosphonic group and the perovskite layer, we attempted a least squares profile refinement of the experimental PDF using the software PDFGui.[48] The starting model was constructed by inserting $\mathrm{C}_{10} \mathrm{PO}_{3}$ chains into the open space between $\mathrm{Sr}_{0.85} \mathrm{Bi}_{0.1} \mathrm{Ta}_{2} \mathrm{O}_{7}$ layers in a similar way as proposed for $\mathrm{n}$-alkylphosphonate derivatives of $\mathrm{HLaNb}_{2} \mathrm{O}_{7} \cdot \mathrm{xH}_{2} \mathrm{O}$.[53] In these materials, it was assumed a monodentate Nb-O-P linkage and a tilt angle of the $n$-alkyl chains of $\sim 57^{\circ}$. Based on the chemical analysis of the $\mathbf{C}_{10} \mathbf{P O}_{3}-\mathrm{HST}$ sample, up to $45 \%$ of $\mathrm{TaO}_{6}$ octahedra could be concerned by a Ta-O-P 
linkage. At the scale of the unit cell, one cannot use partial occupancy parameters to handle such a partial grafting onto $\mathrm{TaO}_{6}$ octahedra, otherwise aberrant close $\mathrm{C}_{10} \mathrm{PO}_{3}$ interatomic distances appear. To circumvent this difficulty, we considered an ordering of the grafted chains on to perovskite layers as depicted in Fig. 6a. In this model, the shortest distance between the carbon atoms from the neighbouring alkyl chains is $\sim 4.4 \AA$ which is consistent with intermolecular distances reported in lipid-layer-like arrangements.[59] We also consider the presence of water molecules close to the tantalum oxide layers, in between phosphonic groups, and forming hydrogen bonds with them.

As can be seen in Fig. 6b, this model performs very well from a very short length scale i.e. within a unit cell for $r$ values below $3.2 \AA$ thus yielding structural information only on local bonding. In this refinement, the constraints of the space group were strictly observed. A stepwise adjustment of the structural parameters of the model was performed (i.e. atomic coordinates, isotropic displacement parameters and one $r$-dependent peak width parameter $r_{\text {cut }}$ ) ensuring that the structural integrity of either components was not destroyed. The refined structural parameters are available in ESI (Fig. S3, Tables S1 and S2). The Ta-O bond lengths in the fragment Ta-O-P and in $\mathrm{Sr}_{0.85} \mathrm{Bi}_{0.1} \mathrm{Ta}_{2} \mathrm{O}_{7}$ cannot be distinguished from each other resulting in a single peak at ca. 2.0 ̊ on the PDF. However, the present structural model implying a grafting of the phosphonate moiety fits well with the PDF data and the refined distances shown in Fig. $6 \mathrm{~b}$ are consistent with a Ta-O-P linkage. This result therefore supports the findings from FTIR and ${ }^{31} \mathrm{P}$ NMR spectroscopy analyses. It is important to note that this structural model featuring $\mathrm{C}_{10} \mathrm{PO}_{3}$-HST as a periodic assembly of $\mathrm{C}_{10} \mathrm{PO}_{3}$ chains arranged as a bilayer between $\mathrm{Sr}_{0.85} \mathrm{Bi}_{0.1} \mathrm{Ta}_{2} \mathrm{O}_{7}$ perovskite blocks fails to reproduce to an acceptable level the atomic correlations above $3.2 \AA$ (Fig. 6c). We attribute this failure mainly to the fact that we did not take into account the variety of bonding interactions in such materials which would require using separate isotropic displacement models to described PDF peak widths.[56] The "freezing" of $\mathrm{C}_{10} \mathrm{PO}_{3}$ units in the interlayer space can also be questioned given the flexibility of long $n$-alkyl chains. Clearly, this level of detail for the structure goes beyond the scope of the present work.

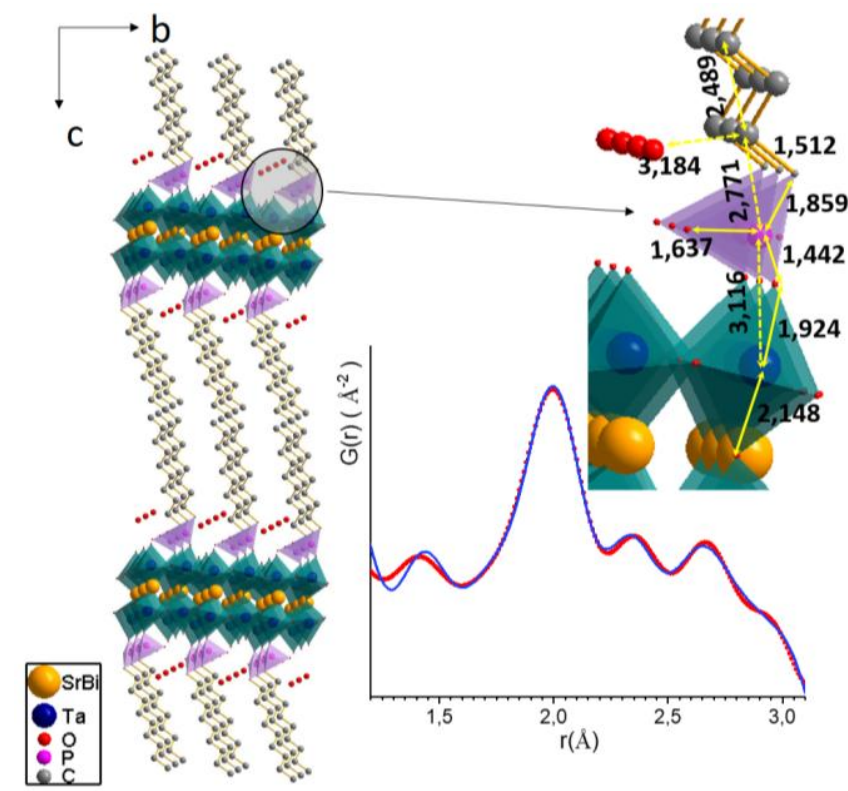

(a)

(b)

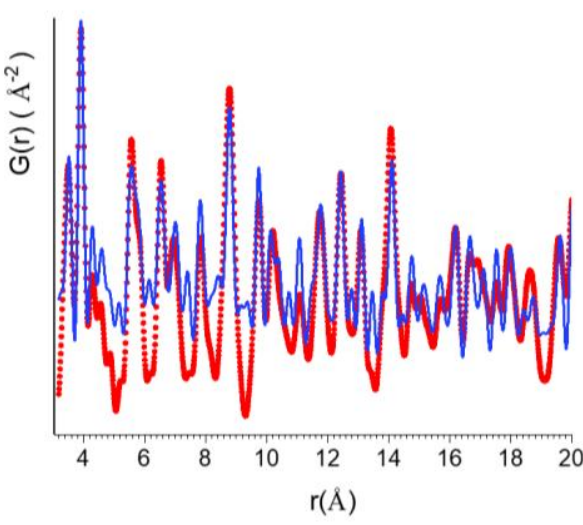

(c)

Fig. 6 (a) Proposed structural arrangement of $\mathrm{C}_{10} \mathrm{PO}_{3}$ chains between $\mathrm{Sr}_{0.85} \mathrm{Bi}_{0.1} \mathrm{Ta}_{2} \mathrm{O}_{7}$ layers for $\mathrm{C}_{10} \mathbf{P O} \mathbf{O}_{3}-\mathbf{H S T}$ sample. (b) Refinement of the structural model in the short range by fitting the experimental PDF of $\mathrm{C}_{10} \mathrm{PO}_{3}$-HST below $3.2 \AA$ : the red points correspond to the experimental PDF and the blue curve represents the calculated PDF $\left(R_{w}\right.$ agreement factor $\left.=8.6 \%\right)$. 
In inset are visualized the refined interatomic distances (in $\AA$ ) consistent with a Ta-O-P linkage. (c) PDF refinement of the structural model in the range from 3 to $20 \AA\left(R_{w}=59.0 \%\right)$

All the same, the PDF results corroborate the conclusions drawn from PXRD, FTIR and NMR analyses and clarify the grafting of phosphonic group onto the $\mathrm{Sr}_{0.85} \mathrm{Bi}_{0.1} \mathrm{Ta}_{2} \mathrm{O}_{7}$ layers, allowing notably to precise the local environment at the interface between the inorganic slab and the organic molecule.

\subsection{Preparation and characterization of functionalized oxide nanosheet dispersions}

As stated in the introduction, exfoliation of charged layered oxides is usually performed by intercalation of bulky cations (typically tetra(n-butyl)ammonium) with a strict control of the cation concentration and subsequent swelling in water till separation of the layers. [20] On the contrary, shear-force-assisted liquid exfoliation is a very efficient method widely used for liquid-phase exfoliation of Van der Waals bonded materials.[60] It consists in applying a shear force on the slabs using a disperser, made of a rotor and a stator, separated by a thin interstice in which the delamination occurs.

Yet, despite its ability to exfoliate in a large scale and even more efficiently than sonication, [61] to our knowledge, this method has very rarely been used for the exfoliation of materials other than graphene and dichalcogenides, [18] and never for the exfoliation of 2D-oxides with perovskite structure.

Actually, as expected, it has not been possible to directly exfoliate HST into a stable suspension using high-shear force disperser. Instead sedimentation occurs almost immediately after shear. Hence no Tyndall effect was observed and light scattering measurements did not allow to get any significant scattered signal. This behavior is explained by the large electrostatic interactions between the charged inorganic layers and interlayer protons, which are too strong to be overcome by external mechanical forces.

On the contrary, the functionalized layered oxide $\mathbf{C}_{10} \mathbf{P O}_{3}$-HST provides a stable turbid solution after shear mixing in isopropanol. Tyndall effect was observed for all supernatants during the selective centrifugation process. Exfoliation parameters (solvent, shear-rate and duration of exfoliation) have been optimized, to provide the highest exfoliation yield and the best stability of suspensions. The solvent has to be polar, since the inorganic layers remain charged, although the charge density has been divided by two by the grafting of decylphosphonate. In addition, by analogy with the work of Coleman et al. on the stabilization of graphene suspensions by adsorbed polymers, [62] we considered solvents with a Hildebrand solubility parameter close to the one of the alkyl chain of the grafted molecule, i.e. decane.[63] With these requirements and after some trials and errors, iso-propanol and acetonitrile appeared as the most suitable solvents. We report in the following the results obtained using iso-propanol, the ones with acetonitrile being similar. Sequential centrifugation was used to separate the nanosheets from non-exfoliated or reaggregated material.[64] In the present case, four centrifugations with increasing speeds between 400 and 1600 rpm were necessary (ascertained by the quality of the DLS correlograms measured on the supernatants), before a final centrifugation at $14000 \mathrm{rpm}$ (18400 g) allows to collect the nanosheets.

The nanosheets were further redispersed in iso-propanol, leading to a stable suspension. Ta and P titration of the obtained suspension by ICP-AES indicated concentrations of $0.126(5)$ and $0.047(6) \mathrm{mmol} \cdot \mathrm{L}^{-1}$ respectively. It is worth underlining here that the $\mathrm{P} /$ Ta ratio value in the nanosheet suspension is almost identical to the one measured for the bulk powder (0.38(6) for the nanosheet suspension, compared to $0.43(4)$ for the bulk). This indicates that the functionalization of the inorganic 
layers remains essentially intact upon the exfoliation and centrifugation procedure, with possibly very moderate ungrafting of the phosphonate ligand.

Assuming consequently that the formula of the nanosheet is the same as the one of the bulk, $\left(\mathrm{C}_{10} \mathrm{H}_{21} \mathrm{PO}_{2}(\mathrm{OH})\right)_{0.9} \mathrm{H}_{1.1} \mathrm{Bi}_{0.1} \mathrm{Sr}_{0.85} \mathrm{Ta}_{2} \mathrm{O}_{7}$, the exfoliation yield can be estimated around $6 \%$. The comparison of the exfoliation yields for different systems investigated by different research groups is somehow difficult due to the more or less narrow size distribution (both in and out of plane) considered and reported (or not).[38] Yet, the present yield is in line for instance with what has been recently reported for the exfoliation by swelling in organic solvent of decorated titanate nanosheets though upon a much shorter time in the present case (1 $\mathrm{h}$ vs. more than $100 \mathrm{~h})$.[36]

\section{Microscopy studies}

The morphology and size of the exfoliated materials were first studied on samples drop-casted on lacey-carbon-coated Cu grids or $\mathrm{Si} / \mathrm{SiO}_{2}$ substrate pre-coated with polyethyleneimine by electron and near field microscopies respectively.

T-SEM studies provide a large scale view of the deposited suspensions, as sheet-like objects (Fig. 7 and S3). From their transparency, the majority of the objects are sheets formed of one to a few layers and can be considered as nanosheets. The electronic contrast (above all in dark field) allows to discriminate the nanomaterials according to their thickness and hence their exfoliation degree (Fig. 7). Nevertheless, some un-exfoliated objects can be observed, despite the selective centrifugation process. It is worth underlining that these objects are very rare compared to the much thinner materials (Fig. S4).

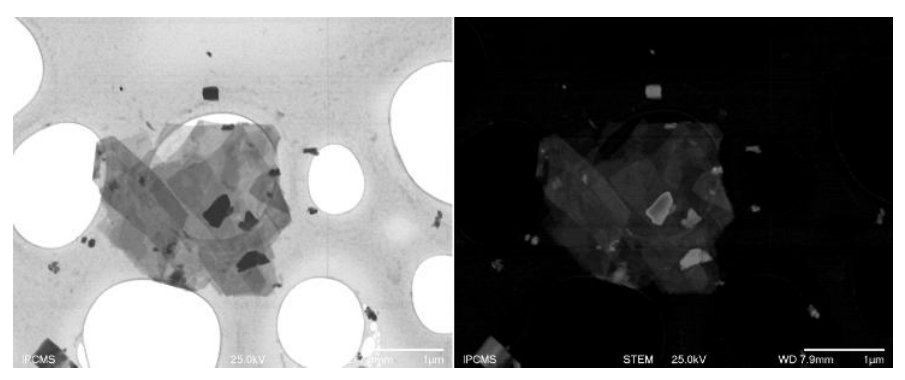

Fig. 7 T-SEM images of nanosheets in bright field (left) and in dark field (right) of exfoliated $\mathbf{C}_{10}$ PO $_{3}$-HST

(the scale bar is $1 \mu \mathrm{m}$ ).

The mean lateral dimensions of the observed thin objects, range between c.a. $300 \mathrm{~nm}$ and c.a. $1 \mu \mathrm{m}$, which is clearly smaller than that of the parent crystals (Fig. S1). Such reduction of size is expected for a high energetic mechanical exfoliation process: the same observation has been made for other materials such as TMDs and for other mechanical exfoliation methods (sonication, micromechanical cleavage).[60] In the case of the exfoliation of zirconium organophosphonate, it has been reported that the use of shear forces enables to obtain particles with larger lateral dimensions than for the ones obtained by ultrasound treatment, even though both methods lead to a reduction of the lateral size compared to the ones observed in the bulk.[61] Such a lateral size reduction has also been observed in the case of "soft" exfoliation, by swelling in organic solvents; the key parameter to control the lateral fracturing being the exfoliation time.[35,65] In our case, the shear rate governs the exfoliation efficiency and fracturing. Higher shear rates and reaction times lead to much smaller particles as already observed in the case of Van der Waals materials.[66] The counter-part of this very fast and efficient exfoliation process is that it is not possible to reduce the exfoliation time to further limit the fracturing while keeping exfoliation rate. Nevertheless, it is worth underlining that the lateral size of the obtained nanosheets remains much larger than the crystalline 
domains of the bulk material, which indicates that the shear-exfoliation process applied to functionalized layered oxides can be considered as soft.

The integrity of the inorganic slab structure was further studied by High Angle Annular Dark Field Scanning Transmission Electron Microscopy (HAADF STEM). Fig. 8 shows HAADF STEM images of a nanosheet. The Fast Fourier Transform of the image in dark field provides the same lattice parameters as the PXRD pattern for the bulk $\mathrm{C}_{10} \mathrm{PO}_{3}$-HST (Table S3). The shear exfoliation process keeps the nanosheet structure intact. It is worth mentioning single layers of hybrid materials are highly sensitive to the electron beam energy. Radiolysis or amorphization of the inorganic slab was observed after repetitive acquisitions or when working at $200 \mathrm{keV}$. Hence all measurements were performed at $100 \mathrm{keV}$.
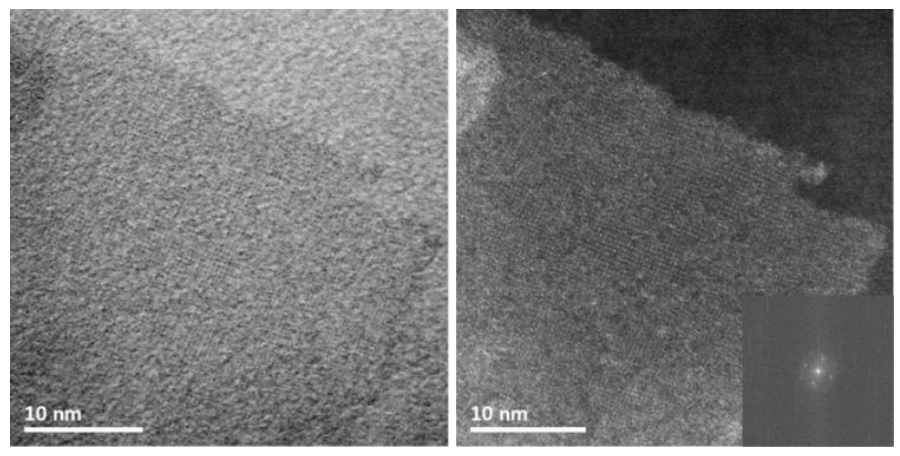

Fig. 8 HAADF STEM image of a $\mathbf{C}_{10} \mathbf{P O}_{3}$-HST monolayer in bright field (left) and dark field (right).

Insert shows the FFT of the dark field image.

As the imaging was performed in HAADF STEM mode, which corresponds to a mass-thickness contrast,[67] the intensity profile can be correlated to the thickness of the materials. The intensity profile of a single monolayer shows a jump of $\sim 1300$ counts (from vacuum to the top of the nanosheet) (Fig. 9). When performed on a stack of layers, series of jumps of $\sim 1000$ counts is observed at each layer step. This slightly smaller value with respect to the one observed from the vacuum to the top of a single monolayer may indicate either a different arrangement of the inserted molecules between two layers compared to what occurs between the first layer and the lacey carbon coated Cu grid[68] or can be due to contamination under the electron beam. Even though it would be tempting to describe the sheet-like objects observed in TEM as monolayers, judging from their apparent transparency, only the intensity profile analysis enables to precise the number of layers involved. From the analysis of the sample on various areas of the TEM grid, the sheets are essentially composed of one to seven layers, either resulting from (partial) exfoliation of the bulk material or from reaggregation of mono layers upon drying (Fig. 9). 

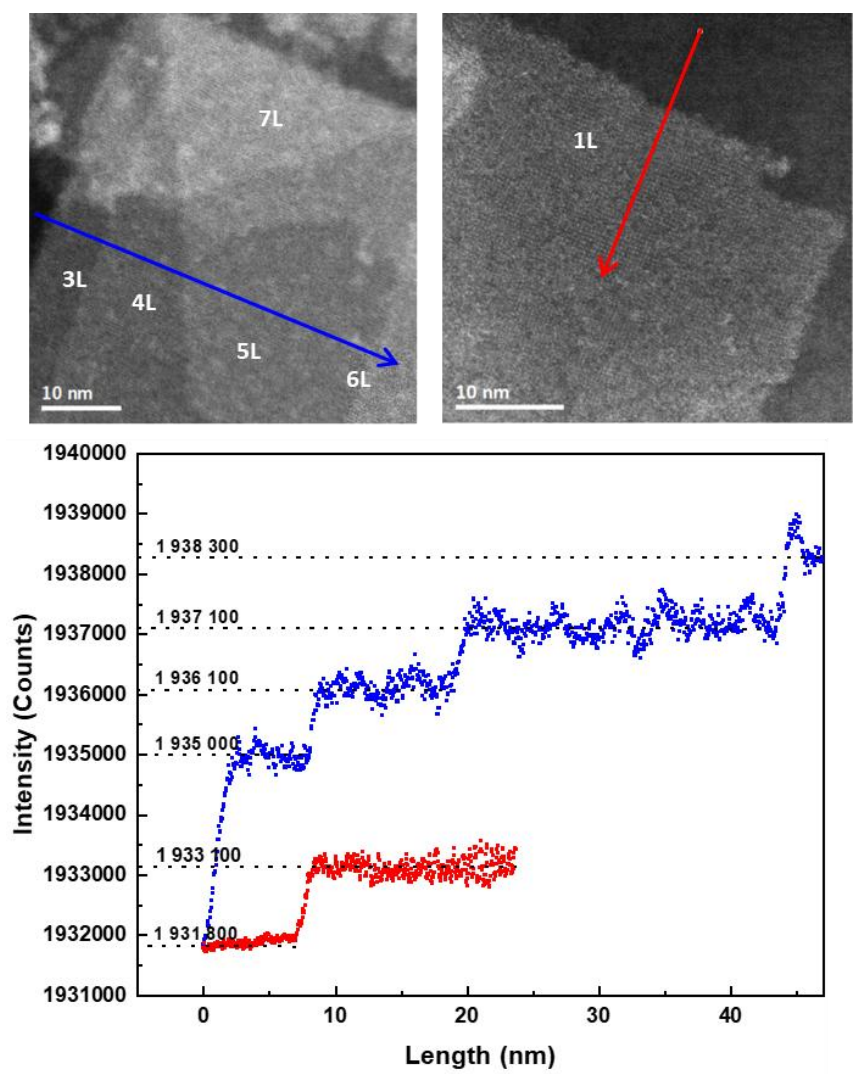

Fig. 9 Dark field clichés of a stacked pile of layers (top left), a monolayer (top right) of exfoliated $\mathbf{C}_{10} \mathbf{P O}_{3}-\mathbf{H S T}$ and intensity profiles (bottom).

EDX analysis performed on the observed nanosheets confirms the presence of $\mathrm{Ta}, \mathrm{Sr}$ and $\mathrm{P}$ in atomic proportion $2.0,1.1$ and 0.88 respectively, in excellent agreement with the ratio determined by ICP-AES on the bulk and on the suspensions (Fig. S5). Although this result does not prove that the anchored molecule remains intact at the surface of the nanosheets, it shows at least that the anchoring phosphonate group is still bonded to the surface and that the in-plane structure is not modified by the shear process.

Finally, AFM studies were performed on a deposit of the nanosheet suspension onto a $\mathrm{SiO}_{2}$ substrate, pre-coated with polyethylene-imine (PEI). Dropcasting followed by evaporation of the solvent appeared to be the most suitable method to deposit large nanosheets. Moreover, even if the charge density of the nanosheets has been considerably lowered by the functionalization, the precoating of the substrate by PEI was necessary to enable adhesion of the nanosheets. Fig. 10 shows an AFM view of functionalized nanosheets (other views are presented in Fig. S6). Given the relatively low concentration of the suspensions due to the necessity to remove non-exfoliated material and to ensure a limited polydispersity, the coverage of the substrate is low. Large objects with a lateral size of about $500 \mathrm{~nm}$ are observed, in accordance with the observations in SEM and TEM and with the DLS characterization of the suspension (see below). The thickness of the nano flakes is around 4 $\mathrm{nm}$, in very good agreement with the expected thickness of one single nanosheet functionalized by decylphosphonic acid (3.4 $\mathrm{nm}$ from XRD of the bulk material). It is worth underlining that no thinner material could be observed. 


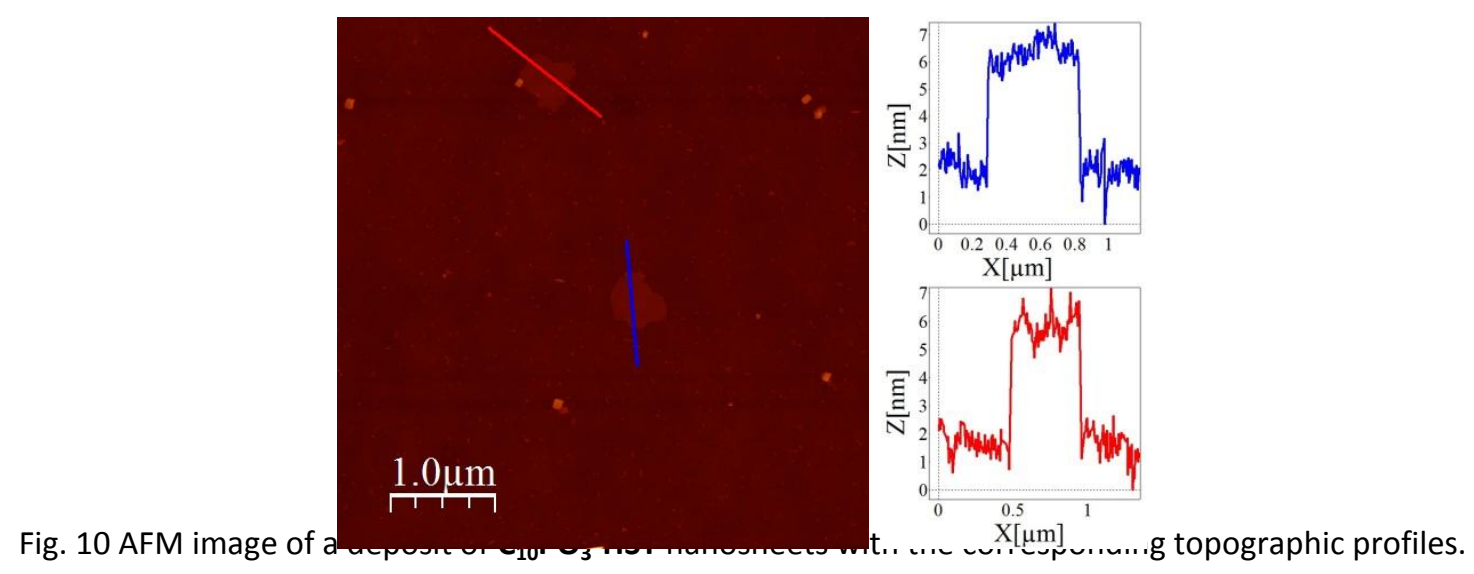

The blue and red profiles on the right correspond to the blue and red lines on the left.

Finally, another minor population of nano objects could be observed in some regions of the subtrates, with a size below $200 \mathrm{~nm}$, the origin of which can be ascribed to partial fragmentation of the nanosheets. [66] The thickness of these smaller flakes takes incremental values, around 4, 7, 10, 13 and $16 \mathrm{~nm}$, as underlined in the representative topographic profiles in Fig. S7 up to $10 \mathrm{~nm}$. These thicknesses are in very good accordance with what is expected for one, two, three, four and five monolayers decorated with decylphosphonic acid, established from the interlamellar distance observed in the bulk material.

\section{Characterization of the nanosheet dispersions}

In the realm of nanomaterials in general, light scattering is commonly used as an efficient and rapid method to get access to the mean hydrodynamic size of nano-objects in solution. Most of its applications are dedicated to spherical nanoparticles with moderate polydispersity. In that case, the diffusion coefficient is expected to be constant and it does not depend on the scattering vector. Consequently, the size determination can be easily performed on a commercial apparatus, with a single angular position for the detector.

Meanwhile, concerning anisotropic shaped materials such as nanosheets or nanoplatelets, the use of light scattering becomes more complicated when one wants to go beyond the simple observation of the Tyndall effect from a suspension. The models become more complex (mostly due to lateral size, thickness and shape distributions) leading to a more time consuming and less straightforward characterization. Coleman et al. characterized graphene and dichalcogenides nanosheets with this technique and derived an empirical relation between their apparent hydrodynamic radius calculated by the StokesEinstein relationship and their size estimated from TEM images. [69]

The group of Philipse et al. performed a complete study on gibbsite platelets.[70] They used a more elaborated model for the diffusion coefficient taking into account the shape of the object, thus considering disks instead of spheres. In the case of gibbsite platelets, they were able to discard the shape distribution issue, and they only had to deal with a limited size polydispersity.

In the present case, all measurements we discuss were conducted on suspensions resulting from a selective size centrifugation process, therefore limiting the polydispersity, essentially in thickness. Moreover, the concentration of the suspension is low (about $0.06 \mathrm{mmol} \cdot \mathrm{L}^{-1}$, or $45 \mathrm{mg} \cdot \mathrm{L}^{-1}$ ), consequently multiple light scattering events are minimized. Scattered 
signals were recorded at several angles (i.e. different scattering vectors) between $20^{\circ}$ and $120^{\circ}$, in order to probe the size and shape dependency.
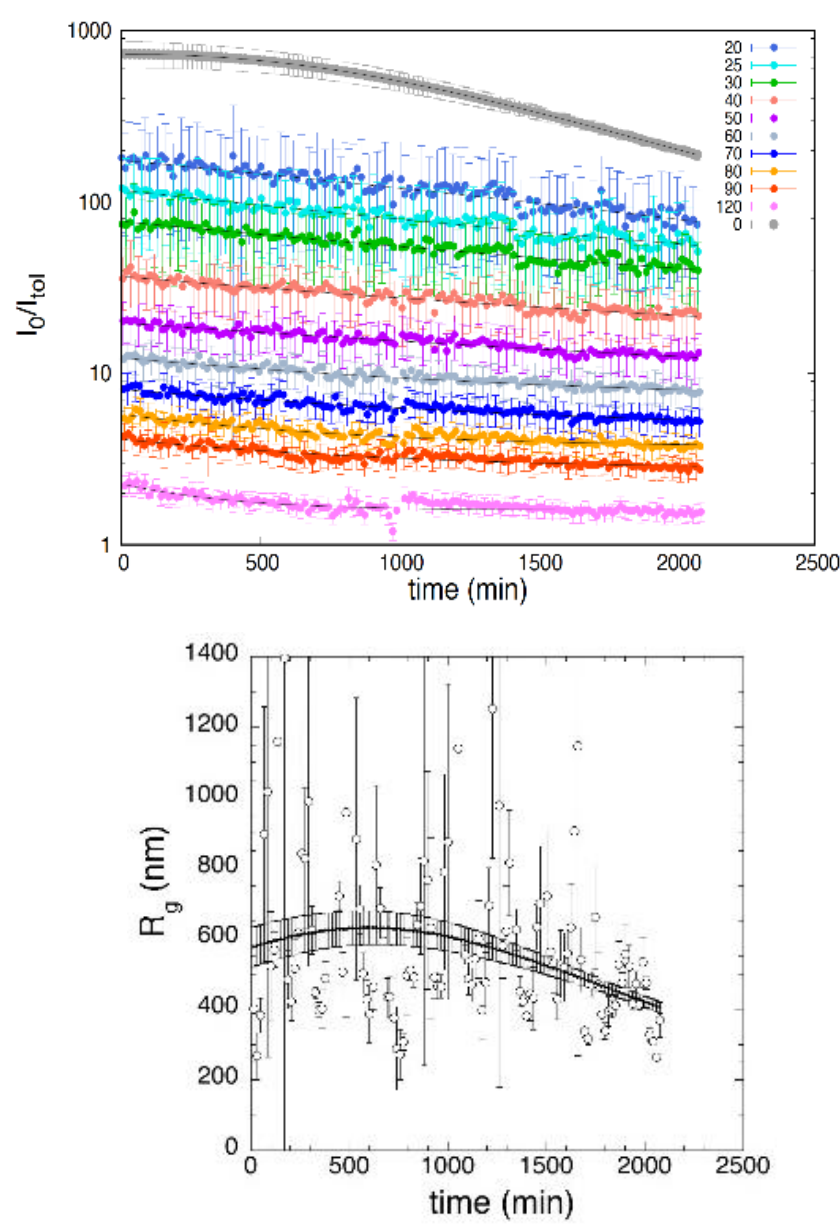

Fig. 11 Top) Scattered light intensity over time for different scattering angles. The continuous lines are interpolating fits. From these fits the scattering intensities at all angles are interpolated at given times and are then used to extrapolate the scattering intensity at zero angle (see SI). Bottom) Time evolution of the apparent z-average radius of gyration: continuous line, values obtained from the interpolating fits in the top figure; open symbols, values obtained with less smoothing interpolating functions (see Supplementary Material).

The characterization of nanosheets in solution implies their colloidal stability which is evidenced in Fig. 11. The scattered intensity at a defined angle remains essentially constant over a time scale of the order of one hour. However, after a few tens of hours, the scattering intensity shows a clear decrease, which is more pronounced at low angles where the contribution of the larger particles is more important. This indicates that some of the larger particles are close to the limits of colloidal stability. On the other hand, the steady decrease of the scattering intensity suggests that the exfoliated platelets do not reaggregate.

As seen in SEM images (Fig. 7 and S3), the $\mathbf{C}_{10} \mathbf{P O}_{3}$-HST nanosheets exhibit both size and shape dispersities, which prevent us from using the form factor of thin disks to analyze the static scattering intensity, as was done for gibbsite platelets. [70] However the measured intensity correlation functions show consistently only one broad mode of relaxation, which extends typically over one decade of relaxation times (see SI). In fact, the relaxation times obtained by CONTIN analysis were in very good agreement with those deduced from the slope at the origin of the field correlation function and we found no 
perturbation by the coupling between the rotational and translational diffusion modes. Therefore, in the following, we will simply analyze the data like for a suspension of Brownian hard spheres and deduce characteristic sizes of our platelets by assimilating them to non-interacting thin disks with a thickness I and a diameter $\mathbf{d}$ with $\mathbf{d}$ >> I. In line with the AFM study (Fig. 10) and the structure of the bulk parent material, we set the thickness at $3 \mathrm{~nm}$, corresponding to a single inorganic slab, functionalized by decylphosphonic molecules.

For each scattering angle, the time evolution of scattering intensity values was fitted to allow the interpolation of these values at given same times for all scattering angles. These interpolated values were then extrapolated to zero scattering vector in the Zimm representation, i.e., $\mathrm{I}^{-1}$ (q) as a function of $\mathrm{q}^{2}$ (see SI for technical details). This allowed to obtain the time evolution of $\mathrm{I}(\mathrm{q}=0)$ (Fig. 11, top) and of the apparent z-averaged radius of gyration $\mathbf{R}_{\mathbf{g}}$ of the platelets (Fig. 11, bottom). It is worth noticing that the use of smoothing interpolating functions reduces greatly the dispersion of the values of $I(q=0)$ and $R_{g}$. The temporal evolutions of both quantities confirm the trend of decrease with time observed for the scattering intensities measured at a given angle. However, the non-monotonous behaviour of the effective radius of gyration might be an artefact linked to the smoothing procedure considering the error bars associated with these values. Using the relationship between the radius of gyration of a thin disk and its dimensions, $\mathbf{R}_{\mathbf{g}}{ }^{2}=\mathbf{d}^{2} / 8+\mathrm{I}^{2} / 12$, [71] we can estimate an apparent diameter of the nanosheets about $1700 \mathrm{~nm}$ at the beginning of the measurement and about $1100 \mathrm{~nm}$ after 35 hours.

The same approach can be used for the analysis of the relaxation times associated with the decay of the intensity correlation functions. Fig. 12 shows the time evolution of the equivalent Stokes-Einstein sphere radius $\mathbf{R}_{\mathbf{h}}$ for two scattering angles. A linear fit through the data for a given angle provides an interpolating function that can be used to generate values at a given time and then extrapolate $\mathbf{R}_{\mathbf{h}}{ }^{-1}$ at zero scattering vector (see SI for technical details). For all scattering angles, the slope of the linear fit was negative thus confirming the trends observed for the scattering intensity and the radius of gyration. Using the relationship $\mathbf{d}=\pi \mathbf{R}_{\mathbf{h}}$, valid for thin disks $\mathbf{I}<\mathbf{d}$,[70] we obtain a second estimation for the apparent diameter of the nanonosheets about $1000 \mathrm{~nm}$ at the beginning of the measurement and about $800 \mathrm{~nm}$ after 35 hours.

To summarize, the light scattering results show that the nanosheets we obtained have an average lateral dimension close to about $1 \mu \mathrm{m}$, i.e., large enough to observe after several hours a sedimentation without relation with a reaggregation of the nanosheets after their exfoliation. Although estimated out of the regular Guinier range and with caveats, this average lateral dimension is in good agreement with the values deduced from the T-SEM and TEM images, taking into account that scattering techniques provide z-averaged dimensions over the size distribution, which tend to be larger than the averages obtained through a limited number of measurements in direct space. 


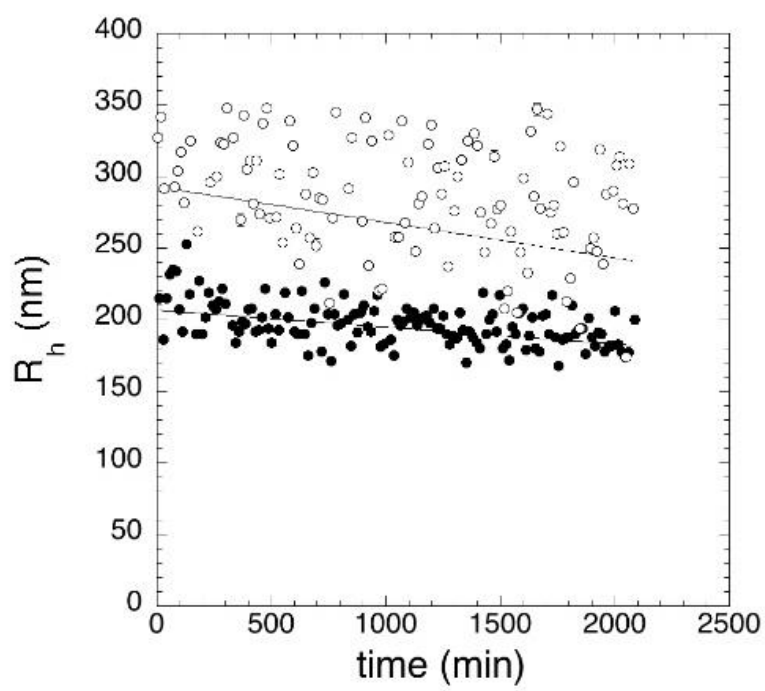

Fig. 12 Decrease with time of the equivalent Stokes-Einstein sphere radius measured for scattering angles $30^{\circ}$ (open symbols) and $90^{\circ}$ (closed symbols). The straight lines correspond to weighted least squares linear fits.

\section{Conclusions}

A rapid and efficient method for the synthesis of functionalized oxide nanosheets has been described, combining microwave-assisted functionalization and liquid-phase exfoliation using shear-force dispersion. A multi technique approach was performed allowing a comprehensive characterization of the pristine hybrid material and its resulting nanosheets.

A new hybrid layered Ta oxide phase with a perovskite structure has been successfully prepared using microwaveassisted reactions. Microwave-assisted functionalization of layered perovskites has emerged recently for amine insertion and alcohol grafting,[39-42] and to the best of our knowledge it is the first time microwaves are employed for functionalization by phosphonic acid derivatives, leading to the desired material in a remarkably short time, much more rapidly than using classical reaction process (3 $\mathrm{h}$ compared to 2 days).[53]

The covalent linkage of the inserted alkyl phosphonic acid has been demonstrated using XRD, IR, solid state NMR and PDF analysis. Notably, PDF allows to unequivocally confirm the monodentate grafting of the phosphonate group, suggested for a related hybrid structure,[53] but never demonstrated so far.

The covalent functionalization gives the possibility to further perform liquid-phase exfoliation of this hybrid material, achieved using high-shear forces. This fast and efficient process leads directly to stable suspensions of covalently functionalized oxide nanosheets and therefore can be viewed as an attractive alternative to the classical two-step approach in which exfoliation is followed by functionalization. $[27,30]$

The "functionalization prior to exfoliation" strategy, developed recently, has been applied up to now to layered Ti, W and Mn oxides functionalized by amines.[37] In the present work, we show that this approach can be generalized to other layered materials, in the very rich family of layered perovskites, and other functionalizations, notably with covalent link between the organic and inorganic moieties which assures the robustness of the functionalization. Furthermore we demonstrate that the prior functionalization decreases the electrostatic stability of the layered perovskite and allows us to considerably accelerate the exfoliation through high shear-force dispersion, as proposed for the exfoliation of layered materials stabilized by weaker 
van der Waals forces.[60] Thus, for the first time, we have obtained a layered Ta oxide with perovskite structure, covalently functionalized by a linear alkyl phosphonic acid that we have been able to exfoliate into nanosheets using shear forces within only $1 \mathrm{~h}$, instead of several tens of hours by swelling in organic solvents.

Using TEM and EDX analyses as well as ICP-AES analyses, direct observation of the nanosheets as deposits and in suspension show clearly that the covalent functionalization of the inorganic layers is entirely preserved upon exfoliation. The characterization of the nanosheets in suspension, although it is often omitted, is crucial for a large scale processing. [69] Here, Static and Dynamic Light Scattering studies enable to determine the mean size of the nanosheets, of about $800 \mathrm{~nm}$.

This rapid and efficient strategy will undoubtedly pave the way for the tunable design of decorated functional nanosheets, through chemical reaction between tethered organic molecules, turning them as single $2 \mathrm{D}$ nanobuilding blocks, enabling for instance the molecular recognition-driven reassembly of different nanosheets, which so far is controlled by electrostatic interactions.[13]

\section{Acknowledgements}

The authors thank the CNRS, the Université de Strasbourg and the Université Clermont Auvergne. The present work benefits from a special funding by the Idex Unistra supported by the Investments for the Future program of the French Government. The Labex NIE is acknowledged for support (ANR-11-LABX-0058-NIE within program ANR-10IDEX-0002-02). The French Ministry for Education and Research is also acknowledged for the Ph-D grant of F. Payet. The MEB-Cro, TEM and XRD facility platforms are acknowledged for access to the SEM, TEM and X-Ray diffractometer. The authors thank Dr. M. Rastei and Dr. M. Pauly for stimulating discussions and D. Burger and C. Kiefer for technical assistance.

\section{CRediT authorship contribution statement}

G. R. designed the research, F. P. synthesized the compounds and performed XRD, FTIR and AFM studies, F. P and C. L. performed SEM studies, C. B. performed the TEM studies, F. L. performed solid state NMR, F. P. and F. S. performed the light diffusion analysis, C. T.-G. carried out the PDF analysis. P. R. and G. R. supervised the research and G. R. wrote the paper from contributions from all authors. All authors discussed the results, commented on the paper and have given approval to the final version of the manuscript.

\section{References}

[1] K.S. Novoselov, A.K. Geim, S.V. Morozov, D. Jiang, Y. Zhang, S.V. Dubonos, I.V. Grigorieva, A.A. Firsov, Electric Field Effect in Atomically Thin Carbon Films, Science. 306 (2004) 666-669. https://doi.org/10.1126/science.1102896.

[2] H. Dong, L. Wu, L. Zhang, H. Chen, C. Gao, Clay nanosheets as charged filler materials for high-performance and foulingresistant thin film nanocomposite membranes, J. Membr. Sci. 494 (2015) 92-103. https://doi.org/10.1016/j.memsci.2015.07.049.

[3] P. Xiong, R. Ma, G. Wang, T. Sasaki, Progress and perspective on two-dimensional unilamellar metal oxide nanosheets and tailored nanostructures from them for electrochemical energy storage, Energy Storage Mater. 19 (2019) $281-298$. https://doi.org/10.1016/j.ensm.2018.12.011.

[4] K. Jiang, P. Xiong, J. Ji, J. Zhu, R. Ma, T. Sasaki, F. Geng, Two-Dimensional Molecular Sheets of Transition Metal Oxides toward Wearable Energy Storage, Acc. Chem. Res. $53 \quad$ (2020) $2443-2455$. https://doi.org/10.1021/acs.accounts.0c00483. 
[5] T.-H. Le, Y. Oh, H. Kim, H. Yoon, Exfoliation of 2D Materials for Energy and Environmental Applications, Chem. - Eur. J. 26 (2020) 6360-6401. https://doi.org/10.1002/chem.202000223.

[6] D.L. Cortie, G.L. Causer, K.C. Rule, H. Fritzsche, W. Kreuzpaintner, F. Klose, Two-Dimensional Magnets: Forgotten History and Recent Progress towards Spintronic Applications, Adv. Funct. Mater. 30 (2020) 1901414. https://doi.org/10.1002/adfm.201901414.

[7] M. Gibertini, M. Koperski, A.F. Morpurgo, K.S. Novoselov, Magnetic 2D materials and heterostructures, Nat. Nanotechnol. 14 (2019) 408-419. https://doi.org/10.1038/s41565-019-0438-6.

[8] C. Tan, X. Cao, X.-J. Wu, Q. He, J. Yang, X. Zhang, J. Chen, W. Zhao, S. Han, G.-H. Nam, M. Sindoro, H. Zhang, Recent Advances in Ultrathin Two-Dimensional Nanomaterials, Chem. Rev. 117 (2017) 6225-6331. https://doi.org/10.1021/acs.chemrev.6b00558.

[9] T. Nakato, J. Kawamata, S. Takagi, eds., Inorganic Nanosheets and Nanosheet-Based Materials, Fundamentals and Applications of Two-Dimensional Systems, Springer Japan, 2017.

[10] M.A. Timmerman, R. Xia, P.T.P. Le, Y. Wang, J.E. ten Elshof, Metal Oxide Nanosheets as 2D Building Blocks for the Design of Novel Materials, Chem. - Eur. J. 26 (2020) 9084-9098. https://doi.org/10.1002/chem.201905735.

[11] D. Sangian, Y. Ide, Y. Bando, A.E. Rowan, Y. Yamauchi, Materials Nanoarchitectonics Using 2D Layered Materials: Recent Developments in the Intercalation Process, Small. 14 (2018) 1800551. https://doi.org/10.1002/smll.201800551.

[12] M. Osada, T. Sasaki, Nanoarchitectonics in dielectric/ferroelectric layered perovskites: from bulk 3D systems to 2D nanosheets, Dalton Trans. 47 (2018) 2841-2851. https://doi.org/10.1039/C7DT03719H.

[13] C. Ziegler, S. Werner, M. Bugnet, M. Wörsching, V. Duppel, G.A. Botton, C. Scheu, B.V. Lotsch, Artificial Solids by Design: Assembly and Electron Microscopy Study of Nanosheet-Derived Heterostructures, Chem. Mater. 25 (2013) 4892-4900. https://doi.org/10.1021/cm402950b.

[14] E. Coronado, C. Martí-Gastaldo, E. Navarro-Moratalla, A. Ribera, S.J. Blundell, P.J. Baker, Coexistence of superconductivity and magnetism by chemical design, Nat. Chem. 2 (2010) 1031-1036. https://doi.org/10.1038/nchem.898.

[15] B.-W. Li, M. Osada, Y. Ebina, S. Ueda, T. Sasaki, Coexistence of Magnetic Order and Ferroelectricity at 2D Nanosheet Interfaces, J. Am. Chem. Soc. 138 (2016) 7621-7625. https://doi.org/10.1021/jacs.6b02722.

[16] T.-H. Gu, J. Kim, S.M. Oh, X. Jin, S.-J. Hwang, Interstratified heterostructures of metal hydroxide nanoclusters and MoS2 monolayers with improved electrode performance, Nanoscale. $12 \quad$ (2020) 11759-11766. https://doi.org/10.1039/DONR02569K.

[17] A.C. Ferrari, F. Bonaccorso, V. Fal'ko, K.S. Novoselov, S. Roche, P. Bøggild, S. Borini, F.H.L. Koppens, V. Palermo, N. Pugno, J.A. Garrido, R. Sordan, A. Bianco, L. Ballerini, M. Prato, E. Lidorikis, J. Kivioja, C. Marinelli, T. Ryhänen, A. Morpurgo, J.N. Coleman, V. Nicolosi, L. Colombo, A. Fert, M. Garcia-Hernandez, A. Bachtold, G.F. Schneider, F. Guinea, C. Dekker, M. Barbone, Z. Sun, C. Galiotis, A.N. Grigorenko, G. Konstantatos, A. Kis, M. Katsnelson, L. Vandersypen, A. Loiseau, V. Morandi, D. Neumaier, E. Treossi, V. Pellegrini, M. Polini, A. Tredicucci, G.M. Williams, B.H. Hong, J.-H. Ahn, J.M. Kim, H. Zirath, B.J. van Wees, H. van der Zant, L. Occhipinti, A.D. Matteo, I.A. Kinloch, T. Seyller, E. Quesnel, X. Feng, K. Teo, N. Rupesinghe, P. Hakonen, S.R.T. Neil, Q. Tannock, T. Löfwander, J. Kinaret, Science and technology roadmap for graphene, related two-dimensional crystals, and hybrid systems, Nanoscale. 7 (2015) 4598-4810. https://doi.org/10.1039/C4NR01600A.

[18] V. Nicolosi, M. Chhowalla, M.G. Kanatzidis, M.S. Strano, J.N. Coleman, Liquid Exfoliation of Layered Materials, Science. 340 (2013) 1226419. https://doi.org/10.1126/science.1226419.

[19] X. Wu, Y. Li, L. Chen, J. Zhao, B. Wu, Z.-B. Zhang, Simultaneous exfoliation and colloidal formation of few-layer semiconducting MoS2 sheets in water, Chem. Commun. 56 (2020) 2035-2038. https://doi.org/10.1039/C9CC08800H.

[20] F. Geng, R. Ma, Y. Ebina, Y. Yamauchi, N. Miyamoto, T. Sasaki, Gigantic Swelling of Inorganic Layered Materials: A Bridge to Molecularly Thin Two-Dimensional Nanosheets, J. Am. Chem. Soc. 136 (2014) 5491-5500. https://doi.org/10.1021/ja501587y.

[21] T. Sasaki, M. Watanabe, H. Hashizume, H. Yamada, H. Nakazawa, Macromolecule-like Aspects for a Colloidal Suspension of an Exfoliated Titanate. Pairwise Association of Nanosheets and Dynamic Reassembling Process Initiated from It, J. Am. Chem. Soc. 118 (1996) 8329-8335. https://doi.org/10.1021/ja960073b.

[22] T.K. Mukhopadhyay, A. Datta, Disentangling the liquid phase exfoliation of two-dimensional materials: an "in silico" perspective, Phys. Chem. Chem. Phys. 22 (2020) 22157-22179. https://doi.org/10.1039/D0CP03128C.

[23] R. Uppuluri, A.S. Gupta, A.S. Rosas, T.E. Mallouk, Soft chemistry of ion-exchangeable layered metal oxides, Chem. Soc. Rev. 47 (2018) 2401-2430. https://doi.org/10.1039/C7CS00290D.

[24] R.E. Schaak, T.E. Mallouk, Perovskites by Design: A Toolbox of Solid-State Reactions, Chem. Mater. 14 (2002) 14551471. https://doi.org/10.1021/cm010689m.

[25] M. Honda, Y. Oaki, H. Imai, Hydrophobic Inorganic-Organic Composite Nanosheets Based on Monolayers of Transition Metal Oxides, Chem. Mater. 26 (2014) 3579-3585. https://doi.org/10.1021/cm5012982. 
[26] Y. Yamamoto, Y. Oaki, H. Imai, Coupled Exfoliation and Surface Functionalization of Titanate Monolayer for Bandgap Engineering, Adv. Mater. Interfaces. 4 (2017) 1601014. https://doi.org/10.1002/admi.201601014.

[27] S. Shori, P.J. Pellechia, H.-C. zur Loye, H.J. Ploehn, Covalent grafting of phenylphosphonate on calcium niobate platelets, J. Colloid Interface Sci. 437 (2015) 97-110. https://doi.org/10.1016/j.jcis.2014.09.024.

[28] S. Akbarian-Tefaghi, T. Rostamzadeh, T.T. Brown, C. Davis-Wheeler, J.B. Wiley, Rapid Exfoliation and Surface Tailoring of Perovskite Nanosheets via Microwave-Assisted Reactions, ChemNanoMat. 3 (2017) 538-550. https://doi.org/10.1002/cnma.201700124.

[29] S. Akbarian-Tefaghi, J.B. Wiley, Microwave-assisted routes for rapid and efficient modification of layered perovskites, Dalton Trans. 47 (2018) 2917-2924. https://doi.org/10.1039/C7DT03865H.

[30] T. Sugaya, M. Ozaki, R. Guégan, N. Idota, Y. Sugahara, Surface Modification of Layered Perovskite Nanosheets with a Phosphorus Coupling Reagent in a Biphasic System, Langmuir. (2019) acs.langmuir.8b03923. https://doi.org/10.1021/acs.langmuir.8b03923.

[31] T. Sugaya, R. Guégan, N. Idota, T. Tsukahara, Y. Sugahara, Highly Efficient Surface Modification of Layered Perovskite Nanosheets with a Phosphorus Coupling Reagent Making Use of Microchannels, Langmuir. 36 (2020) 7252-7258. https://doi.org/10.1021/acs.langmuir.0c00560.

[32] N. Kimura, Y. Kato, R. Suzuki, A. Shimada, S. Tahara, T. Nakato, K. Matsukawa, P.H. Mutin, Y. Sugahara, Single- and Double-Layered Organically Modified Nanosheets by Selective Interlayer Grafting and Exfoliation of Layered Potassium Hexaniobate, Langmuir. 30 (2014) 1169-1175. https://doi.org/10.1021/la404223x.

[33] Y. Asai, Y. Ariake, H. Saito, N. Idota, K. Matsukawa, T. Nishino, Y. Sugahara, Layered perovskite nanosheets bearing fluoroalkoxy groups: their preparation and application in epoxy-based hybrids, RSC Adv. 4 (2014) 26932-26939. https://doi.org/10.1039/C4RA01777C.

[34] H. Matsui, Y. Oaki, H. Imai, Surface-functionalized hydrophilic monolayer of titanate and its application for dopamine detection, Chem. Commun. 52 (2016) 9466-9469. https://doi.org/10.1039/C6CC02940J.

[35] M. Honda, Y. Oaki, H. Imai, Hydrophobic monolayered nanoflakes of tungsten oxide: coupled exfoliation and fracture in a nonpolar organic medium, Chem. Commun. 51 (2015) 10046-10049. https://doi.org/10.1039/C5CC02203G.

[36] G. Nakada, H. Imai, Y. Oaki, Few-layered titanate nanosheets with large lateral size and surface functionalization: potential for the controlled exfoliation of inorganic-organic layered composites, Chem. Commun. 54 (2018) $244-247$. https://doi.org/10.1039/C7CC07759A.

[37] Y. Oaki, Exfoliation Chemistry of Soft Layered Materials toward Tailored 2D Materials, Chem. Lett. 50 (2021) $305-315$. https://doi.org/10.1246/cl.200747.

[38] G. Nakada, Y. Igarashi, H. Imai, Y. Oaki, Materials-Informatics-Assisted High-Yield Synthesis of 2D Nanomaterials through Exfoliation, Adv. Theory Simul. 2 (2019) 1800180. https://doi.org/10.1002/adts.201800180.

[39] J.R. Boykin, L.J. Smith, Rapid Microwave-Assisted Grafting of Layered Perovskites with n-Alcohols, Inorg. Chem. 54 (2015) 4177-4179. https://doi.org/10.1021/ic503001w.

[40] S. Akbarian-Tefaghi, E. Teixeira Veiga, G. Amand, J.B. Wiley, Rapid Topochemical Modification of Layered Perovskites via Microwave Reactions, Inorg. Chem. 55 (2016) 1604-1612. https://doi.org/10.1021/acs.inorgchem.5b02514.

[41] Y. Wang, E. Delahaye, C. Leuvrey, F. Leroux, P. Rabu, G. Rogez, Efficient Microwave-Assisted Functionalization of the Aurivillius-Phase Bi2SrTa2O9, Inorg. Chem. 55 (2016) 4039-4046. https://doi.org/10.1021/acs.inorgchem.6b00338.

[42] Y. Wang, M. Nikolopoulou, E. Delahaye, C. Leuvrey, F. Leroux, P. Rabu, G. Rogez, Microwave-assisted functionalization of the Aurivillius phase Bi2SrTa2O9: diol grafting and amine insertion vs. alcohol grafting, Chem. Sci. 9 (2018) 71047114. https://doi.org/10.1039/C8SC01754A.

[43] Y. Wang, C. Leuvrey, E. Delahaye, F. Leroux, P. Rabu, C. Taviot-Guého, G. Rogez, Tuning the organization of the interlayer organic moiety in a hybrid layered perovskite, J. Solid State Chem. 269 (2019) 532-539. https://doi.org/10.1016/j.jssc.2018.10.034.

[44] Y. Tsunoda, M. Shirata, W. Sugimoto, Z. Liu, O. Terasaki, K. Kuroda, Y. Sugahara, Preparation and HREM Characterization of a Protonated Form of a Layered Perovskite Tantalate from an Aurivillius Phase Bi2SrTa2O9 via Acid Treatment, Inorg. Chem. 40 (2001) 5768-5771. https://doi.org/10.1021/ic010266m.

[45] S. Ida, C. Ogata, U. Unal, K. Izawa, T. Inoue, O. Altuntasoglu, Y. Matsumoto, Preparation of a Blue Luminescent Nanosheet Derived from Layered Perovskite Bi2SrTa209, J. Am. Chem. Soc. 129 (2007) 8956-8957. https://doi.org/10.1021/ja073105b.

[46] Z. Peng, X. Xing, X. Chen, Preparation and structure of a new layered organic-inorganic hybrid between the protonated form of a perovskite Bi2SrTa2O9 and tetraphenylporphyrin by the intercalation behavior, J. Alloys Compd. 425 (2006) 323-328. https://doi.org/10.1016/j.jallcom.2006.01.051.

[47] P. Juhás, T. Davis, C.L. Farrow, S.J.L. Billinge, PDFgetX3: a rapid and highly automatable program for processing powder diffraction data into total scattering pair distribution functions, J. Appl. Crystallogr. 46 (2013) 560-566. https://doi.org/10.1107/S0021889813005190. 
[48] C.L. Farrow, P. Juhas, J.W. Liu, D. Bryndin, E.S. Božin, J. Bloch, T. Proffen, S.J.L. Billinge, PDFfit2 and PDFgui: computer programs for studying nanostructure in crystals, J. Phys. Condens. Matter. 19 (2007) 335219. https://doi.org/10.1088/0953-8984/19/33/335219.

[49] D.B. Williams, C.B. Carter, in: Transm. Electron Microsc. Part 1 Basics, Springer Science, Business Media, New York, 2009: pp. 64-68.

[50] S.W. Provencher, A constrained regularization method for inverting data represented by linear algebraic or integral equations, Comput. Phys. Commun. 27 (1982) 213-227. https://doi.org/10.1016/0010-4655(82)90173-4.

[51] S.W. Provencher, CONTIN: A general purpose constrained regularization program for inverting noisy linear algebraic and integral equations, Comput. Phys. Commun. 27 (1982) 229-242. https://doi.org/10.1016/0010-4655(82)90174-6.

[52] I. Echavarri Franco, P. Lorchat, J.-P. Lamps, M. Schmutz, A. Schröder, J.-M. Catala, J. Combet, F. Schosseler, From Chain Collapse to New Structures: Spectroscopic Properties of Poly(3-thiophene acetic acid) upon Binding by Alkyl Trimethylammonium Bromide Surfactants, Langmuir. 28 (2012) 4815-4828. https://doi.org/10.1021/la204862u.

[53] A. Shimada, Y. Yoneyama, S. Tahara, P.H. Mutin, Y. Sugahara, Interlayer surface modification of the protonated ionexchangeable layered perovskite HLaNb2O7•xH2O with organophosphonic acids, Chem. Mater. 21 (2009) $4155-4162$. https://doi.org/10.1021/cm900228c.

[54] G. Guerrero, P.H. Mutin, A. Vioux, Anchoring of Phosphonate and Phosphinate Coupling Molecules on Titania Particles, Chem. Mater. 13 (2001) 4367-4373. https://doi.org/10.1021/cm001253u.

[55] K.R. Whittle, N.C. Hyatt, I.M. Reaney, Phase Transitions in Lanthanum-Doped Strontium Bismuth Tantalate, Chem. Mater. 20 (2008) 6427-6433. https://doi.org/10.1021/cm800475z.

[56] D. Prill, P. Juhás, M.U. Schmidt, S.J.L. Billinge, Modelling pair distribution functions (PDFs) of organic compounds: describing both intra- and intermolecular correlation functions in calculated PDFs, J. Appl. Crystallogr. 48 (2015) 171178. https://doi.org/10.1107/S1600576714026454.

[57] V. Petkov, V. Parvanov, P. Trikalitis, C. Malliakas, T. Vogt, M.G. Kanatzidis, Three-Dimensional Structure of Nanocomposites from Atomic Pair Distribution Function Analysis: Study of Polyaniline and (Polyaniline)0.5V2O5·1.0H2O, J. Am. Chem. Soc. 127 (2005) 8805-8812. https://doi.org/10.1021/ja051315n.

[58] T. Proffen, S.J.L. Billinge, PDFFIT, a program for full profile structural refinement of the atomic pair distribution function, J. Appl. Crystallogr. 32 (1999) 572-575. https://doi.org/10.1107/S0021889899003532.

[59] D. Boczula, A. Cały, D. Dobrzyńska, J. Janczak, J. Zoń, Structural and vibrational characteristics of amphiphilic phosphonate salts, J. Mol. Struct. 1007 (2012) 220-226. https://doi.org/10.1016/j.molstruc.2011.10.051.

[60] J.N. Coleman, M. Lotya, A. O’Neill, S.D. Bergin, P.J. King, U. Khan, K. Young, A. Gaucher, S. De, R.J. Smith, I.V. Shvets, S.K. Arora, G. Stanton, H.-Y. Kim, K. Lee, G.T. Kim, G.S. Duesberg, T. Hallam, J.J. Boland, J.J. Wang, J.F. Donegan, J.C. Grunlan, G. Moriarty, A. Shmeliov, R.J. Nicholls, J.M. Perkins, E.M. Grieveson, K. Theuwissen, D.W. McComb, P.D. Nellist, V. Nicolosi, Two-Dimensional Nanosheets Produced by Liquid Exfoliation of Layered Materials, Science. 331 (2011) 568-571. https://doi.org/10.1126/science.1194975.

[61] K. Kopecká, K. Melánová, L. Beneš, P. Knotek, M. Mazur, V. Zima, Exfoliation of layered mixed zirconium 4sulfophenylphosphonate phenylphosphonates, Dalton Trans. $49 \quad$ (2020) 3816-3823. https://doi.org/10.1039/C9DT03883C.

[62] P. May, U. Khan, J.M. Hughes, J.N. Coleman, Role of Solubility Parameters in Understanding the Steric Stabilization of Exfoliated Two-Dimensional Nanosheets by Adsorbed Polymers, J. Phys. Chem. C. 116 (2012) 11393-11400. https://doi.org/10.1021/jp302365w.

[63] A.F.M. Barton, Handbook of solubility parameters and other cohesion parameters, 2nd ed., CRC Press, 1991.

[64] S.P. Ogilvie, M.J. Large, M.A. O'Mara, P.J. Lynch, C.L. Lee, A.A.K. King, C. Backes, A.B. Dalton, Size selection of liquidexfoliated 2D nanosheets, 2D Mater. 6 (2019) 031002. https://doi.org/10.1088/2053-1583/ab0dc3.

[65] R. Mizuguchi, H. Imai, Y. Oaki, Formation processes, size changes, and properties of nanosheets derived from exfoliation of soft layered inorganic-organic composites, Nanoscale Adv. 2 (2020) 1168-1176. https://doi.org/10.1039/D0NA00084A.

[66] A. Liscio, K. Kouroupis-Agalou, X.D. Betriu, A. Kovtun, E. Treossi, N.M. Pugno, G.D. Luca, L. Giorgini, V. Palermo, Evolution of the size and shape of 2D nanosheets during ultrasonic fragmentation, 2D Mater. 4 (2017) 025017. https://doi.org/10.1088/2053-1583/aa57ff.

[67] S.J. Pennycook, Z-Contrast Transmission Electron Microscopy: Direct Atomic Imaging of Materials, Annu. Rev. Mater. Sci. 22 (1992) 171-195. https://doi.org/10.1146/annurev.ms.22.080192.001131.

[68] K.S. Virdi, Y. Kauffmann, C. Ziegler, P. Ganter, P. Blaha, B.V. Lotsch, W.D. Kaplan, C. Scheu, Band Gap Extraction from Individual Two-Dimensional Perovskite Nanosheets Using Valence Electron Energy Loss Spectroscopy, J. Phys. Chem. C. 120 (2016) 11170-11179. https://doi.org/10.1021/acs.jpcc.6b00142.

[69] M. Lotya, A. Rakovich, J.F. Donegan, J.N. Coleman, Measuring the lateral size of liquid-exfoliated nanosheets with dynamic light scattering, Nanotechnology. 24 (2013) 265703. https://doi.org/10.1088/0957-4484/24/26/265703. 
[70] F.M. van der Kooij, A.P. Philipse, J.K.G. Dhont, Sedimentation and Diffusion in Suspensions of Sterically Stabilized Colloidal Platelets, Langmuir. 16 (2000) 5317-5323. https://doi.org/10.1021/la991571b.

[71] A. Guinier, G. Fournet, Small-angle scattering of X-rays, Wiley, New-York, 1955. 


\section{Figure captions}

Fig. 1 XRD patterns of $\mathbf{H S T}$ (red), $\mathbf{C}_{4} \mathbf{N}-\mathbf{H S T}$ (cyan) and $\mathbf{C}_{10} \mathrm{PO}_{3}$-HST (black).

Fig. 2 Infrared spectra of HST (red), $\mathbf{C}_{4} \mathbf{N}$-HST (cyan), $\mathbf{C}_{10} \mathrm{PO}_{3}-\mathrm{HST}$ (black) and 1-decylphosphonic acid (blue)

Fig. $3{ }^{13} \mathrm{C} \mathrm{CP} / \mathrm{MAS} N M R$ spectrum of $\mathrm{C}_{10} \mathrm{PO}_{3}$-HST (the asterisk is attributed to the presence of an impurity of butylamine)

Fig. 4 (left) Experimental PDF for $\mathbf{C}_{10}$ PO $_{3}$-HST sample obtained up to $200 \AA$ and (right) details in the range from 1 to $40 \AA$

Fig. 5 (a) Experimental PDF for $\mathbf{C}_{10} \mathbf{P O}_{3}$-HST (bottom) compared to the model PDF for " $\mathrm{Sr}_{0.85} \mathrm{Bi}_{0.1} \mathrm{Ta}_{2} \mathrm{O}_{7}$ " perovskite layers (middle) and the experimental PDF for 1-decylphosphonic acid (top). Distances between nearest atoms (b) along the decylphosphonic chain and (c) in $\mathrm{Sr}_{0.85} \mathrm{Bi}_{0.1} \mathrm{Ta}_{2} \mathrm{O}_{7}$ perovskite layer model

Fig. 6 (a) Proposed structural arrangement of $\mathrm{C}_{10} \mathrm{PO}_{3}$ chains between $\mathrm{Sr}_{0.85} \mathrm{Bi}_{0.1} \mathrm{Ta}_{2} \mathrm{O}_{7}$ layers for $\mathrm{C}_{10} \mathrm{PO}-\mathrm{HST}$ sample. (b) Refinement of the structural model in the short range by fitting the experimental PDF of $\mathbf{C}_{10}$ PO $_{3}$-HST below $3.2 \AA$ : the red points correspond to the experimental PDF and the blue curve represents the calculated PDF $\left(R_{w}\right.$ agreement factor $\left.=8.6 \%\right)$. In inset are visualized the refined interatomic distances (in $\AA$ ) consistent with a Ta-O-P linkage. (c) PDF refinement of the structural model in the range from 3 to $20 \AA\left(R_{w}=59.0 \%\right)$

Fig. 7 T-SEM images of nanosheets in bright field (left) and in dark field (right) of exfoliated $\mathbf{C}_{10}$ PO $\mathbf{O}_{3}$-HST (the scale bar is 1 $\mu \mathrm{m})$.

Fig. 8 HAADF STEM image of a $\mathbf{C}_{10} \mathbf{P O}_{3}$-HST monolayer in bright field (left) and dark field (right). Insert shows the FFT of the dark field image.

Fig. 9 Dark field clichés of a stacked pile of layers (top left), a monolayer (top right) of exfoliated $\mathbf{C}_{10} \mathbf{P O}-\mathbf{H S T}_{3}$ and intensity profiles (bottom).

Fig. 10 AFM image of a deposit of $\mathbf{C}_{10} \mathbf{P O}_{3}$-HST nanosheets with the corresponding topographic profiles.

The blue and red profiles on the right correspond to the blue and red lines on the left.

Fig. 11 Top) Scattered light intensity over time for different scattering angles. The continuous lines are interpolating fits. From these fits the scattering intensities at all angles are interpolated at given times and are then used to extrapolate the scattering intensity at zero angle (see SI). Bottom) Time evolution of the apparent z-average radius of gyration: continuous line, values obtained from the interpolating fits in the top figure; open symbols, values obtained with less smoothing interpolating functions (see Supplementary Material).

Fig. 12 Decrease with time of the equivalent Stokes-Einstein sphere radius measured for scattering angles $30^{\circ}$ (open symbols) and $90^{\circ}$ (closed symbols). The straight lines correspond to weighted least squares linear fits. 


\section{Supplementary Material}

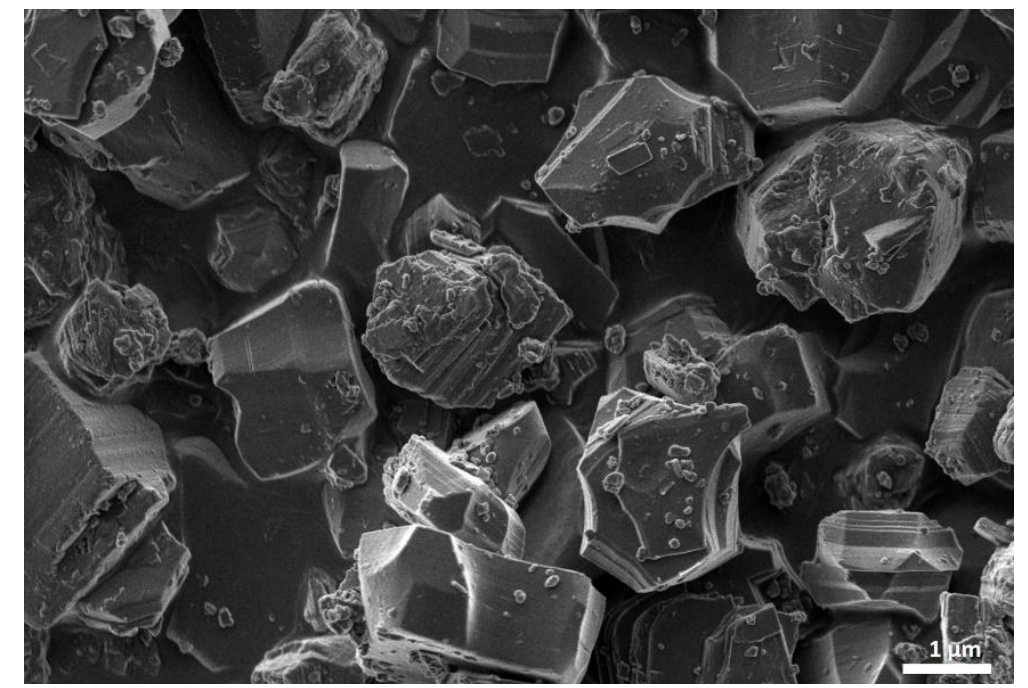

Figure S1. SEM image of bulk $\mathbf{C}_{10} \mathrm{PO}_{3}$ - $\mathrm{HST}$ (scale bar is $1 \mu \mathrm{m}$ ).

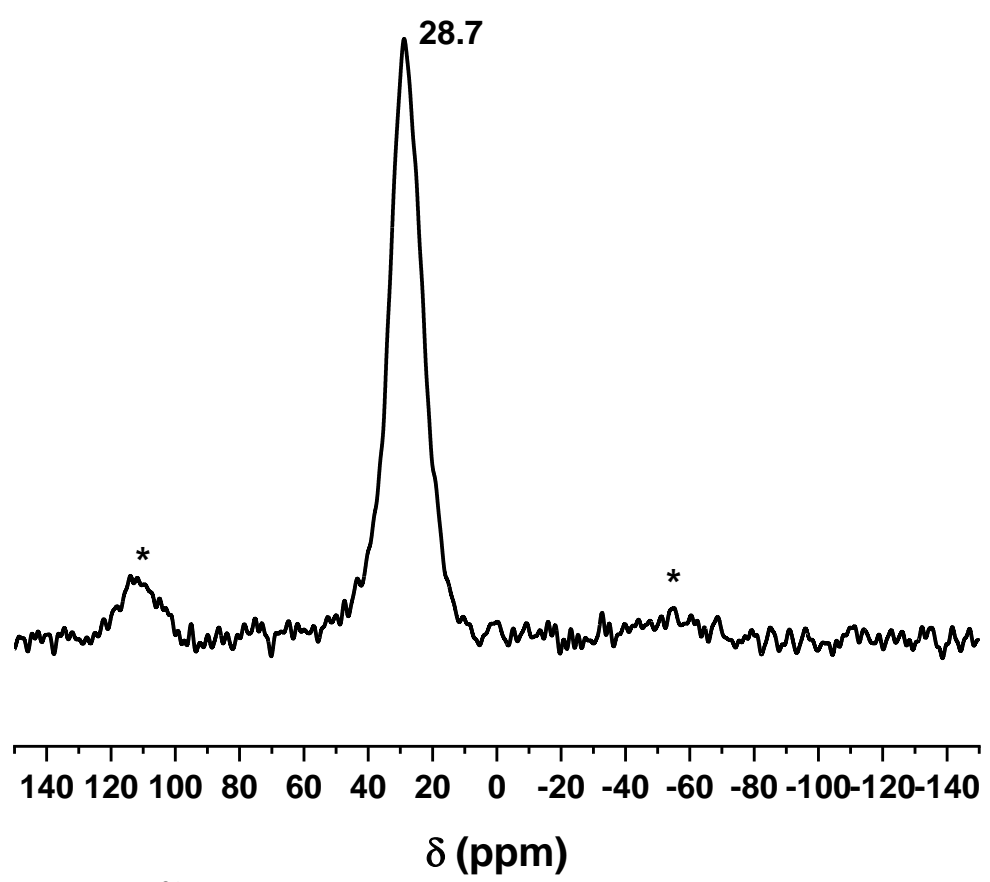

Figure S2. ${ }^{31} \mathrm{P}$ MAS NMR spectrum of $\mathbf{C}_{10} \mathrm{PO}_{3}-\mathrm{HST}$ (the signals marked with asterisks are attributed to spinning sidebands). 


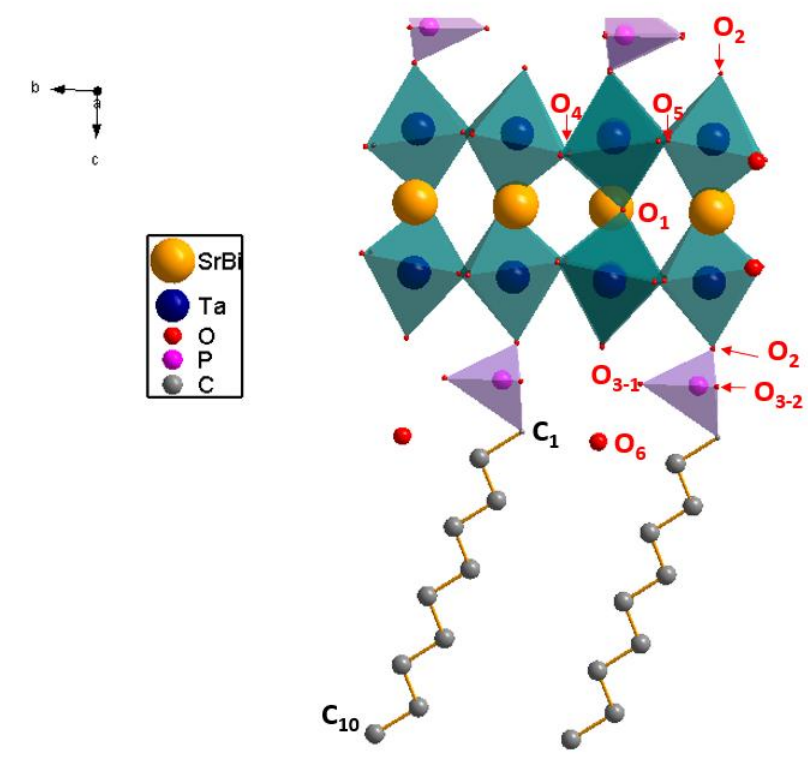

Figure S3. Model structure of $\mathbf{C}_{10} \mathbf{P O}_{3}-\mathrm{HST}$. The atoms of the perovskite block are named by reference to the model structure reported by Whittle et al..[1]

Table S1. Experimental details and instrumental parameters for the refinement of the PDF of $\mathbf{C}_{10} \mathrm{PO}_{3}-\mathrm{HST}_{\text {in }}$ the $1.2-3.2 \AA$ range.

\begin{tabular}{|l|l|}
\hline$\lambda(\AA)$ Silver $\mathrm{K}_{\alpha 1 / \alpha 2}$ & $\lambda_{\alpha 1}=0.559421 \AA ; \lambda_{\alpha 2}=0.563812 \AA$ \\
\hline $\mathrm{Q}_{\max \text { ins }}\left(\AA^{-1}\right)$ & 21.5 \\
\hline $\mathrm{Q}_{\max }\left(\AA^{-1}\right)$ & 21 \\
\hline $\mathrm{Q}_{\text {damp }}\left(\AA^{-1}\right)$ & 0.01 \\
\hline Unit cell $\quad(\AA)$ & $\mathrm{a}=5.52715(9)$ \\
orthorhombic & $\mathrm{b}=5.55459(12)$ \\
$\mathrm{A} 2_{1}$ am & $\mathrm{c}=65.2$ \\
\hline $\mathrm{r}_{\text {cut }}(\AA)$ & 5.2 \\
\hline $\mathrm{S}_{\text {rat }}$ & $0.0218(6)$ \\
\hline $\mathrm{R}_{\mathrm{w}}$ & 0.086 \\
\hline
\end{tabular}

Table S2. Results of the refinement of the PDF of $\mathbf{C}_{10} \mathbf{P O}_{3}-\mathbf{H S T}$ in the 1.2-3.2 $\mathrm{A} r$ range. Errors are not reported for the refined parameters as the standard deviations are not properly calculated by PDFgui (standard data reduction obtained by PDFgetX3 does not include the standard deviations of the experimental PDF, which is needed to properly calculate the errors).[2]

\begin{tabular}{|c|c|c|c|c|c|c|}
\hline \multicolumn{7}{|c|}{ Atomic positions in the orthorhombic cell (SG A2 ${ }_{1}$ am) } \\
\hline & Wyckoff & $x$ & $y$ & $z$ & $\operatorname{ADP}\left(\AA^{2}\right)^{a}$ & $\mathrm{SOF}^{\mathrm{b}}$ \\
\hline $\mathrm{Sr}$ & $4 a$ & 0.06019 & 0.24357 & 0 & 0.0040 & 0.85 \\
\hline $\mathrm{Bi}$ & $4 a$ & 0.56019 & 0.25640 & 0.5 & 0.0040 & 0.1 \\
\hline $\mathrm{Ta}$ & $8 \mathrm{~b}$ & 0.53501 & 0.74631 & 0.46755 & 0.0002 & 1.0 \\
\hline $\mathrm{O}_{1}$ & $4 a$ & 0.06530 & 0.69359 & 0 & 0.0100 & 1.0 \\
\hline $\mathrm{O}_{2}$ & $8 \mathrm{~b}$ & 0.50866 & 0.71750 & 0.43824 & 0.0040 & 1.0 \\
\hline $\mathrm{O}_{4}$ & $8 b$ & 0.78308 & 0.97928 & 0.02393 & 0.0040 & 1.0 \\
\hline $\mathrm{O}_{5}$ & $8 \mathrm{~b}$ & 0.79971 & 0.97584 & 0.53186 & 0.0002 & 1.0 \\
\hline$P$ & $8 b^{c}$ & $\begin{array}{l}0.15708 \\
0.65708 \\
0.15708 \\
0.65708 \\
\end{array}$ & $\begin{array}{l}0.21339 \\
0.78660 \\
0.71339 \\
0.28660 \\
\end{array}$ & $\begin{array}{l}0.42102 \\
0.57897 \\
0.92102 \\
0.07897\end{array}$ & $\begin{array}{l}0.0030 \\
0.0030 \\
0.0030 \\
0.0030 \\
\end{array}$ & $\begin{array}{l}0.9 \\
0.9 \\
0.9 \\
0.9 \\
\end{array}$ \\
\hline $\mathrm{O}_{3-1}$ & $8 b^{c}$ & $\begin{array}{l}0.15655 \\
0.65655 \\
0.15655 \\
0.65655\end{array}$ & $\begin{array}{l}0.91868 \\
0.08132 \\
0.41868 \\
0.58132\end{array}$ & $\begin{array}{l}0.42111 \\
0.57888 \\
0.92111 \\
0.07888\end{array}$ & $\begin{array}{l}0.0003 \\
0.0003 \\
0.0003 \\
0.0003\end{array}$ & $\begin{array}{l}0.9 \\
0.9 \\
0.9 \\
0.9\end{array}$ \\
\hline $\mathrm{O}_{3-2}$ & $8 b^{c}$ & $\begin{array}{l}0.43761 \\
0.93761 \\
0.43761\end{array}$ & $\begin{array}{l}0.30855 \\
0.69144 \\
0.80855\end{array}$ & $\begin{array}{l}0.42127 \\
0.57872 \\
0.92127\end{array}$ & $\begin{array}{l}0.0110 \\
0.0110 \\
0.0110\end{array}$ & $\begin{array}{l}0.9 \\
0.9 \\
0.9\end{array}$ \\
\hline
\end{tabular}




\begin{tabular}{|c|c|c|c|c|c|c|}
\hline & & 0.93761 & 0.19144 & 0.07872 & 0.0110 & 0.9 \\
\hline $\mathrm{O}_{6}\left(\mathrm{H}_{2} \mathrm{O}\right)$ & $8 b^{c}$ & $\begin{array}{l}0.50720 \\
0.00720 \\
0.50720 \\
0.00720\end{array}$ & $\begin{array}{l}0.71177 \\
0.28822 \\
0.21177 \\
0.78822\end{array}$ & $\begin{array}{l}0.39351 \\
0.60648 \\
0.89351 \\
0.10648\end{array}$ & $\begin{array}{l}0.0006 \\
0.0006 \\
0.0006 \\
0.0006\end{array}$ & $\begin{array}{l}1.0 \\
1.0 \\
1.0 \\
1.0\end{array}$ \\
\hline $\mathrm{C}_{1}$ & $8 b^{c}$ & $\begin{array}{l}0.99618 \\
0.49618 \\
0.99618 \\
0.49618\end{array}$ & $\begin{array}{l}0.32121 \\
0.67879 \\
0.82121 \\
0.17879\end{array}$ & $\begin{array}{l}0.39773 \\
0.60226 \\
0.89773 \\
0.10226\end{array}$ & $\begin{array}{l}0.0001 \\
0.0001 \\
0.0001 \\
0.0001\end{array}$ & $\begin{array}{l}0.9 \\
0.9 \\
0.9 \\
0.9 \\
\end{array}$ \\
\hline$C_{2}$ & $8 b^{c}$ & $\begin{array}{l}0.91004 \\
0.41004 \\
0.91004 \\
0.41004\end{array}$ & $\begin{array}{l}0.10944 \\
0.89055 \\
0.60944 \\
0.39055\end{array}$ & $\begin{array}{l}0.38512 \\
0.61487 \\
0.88512 \\
0.11487\end{array}$ & $\begin{array}{l}0.0001 \\
0.0001 \\
0.0001 \\
0.0001\end{array}$ & $\begin{array}{l}0.9 \\
0.9 \\
0.9 \\
0.9\end{array}$ \\
\hline$C_{3}$ & $8 b^{c}$ & $\begin{array}{l}0.77848 \\
0.27848 \\
0.77848 \\
0.27848 \\
\end{array}$ & $\begin{array}{l}0.20281 \\
0.79718 \\
0.70281 \\
0.29718 \\
\end{array}$ & $\begin{array}{l}0.36622 \\
0.63377 \\
0.86622 \\
0.13377\end{array}$ & $\begin{array}{l}0.0001 \\
0.0001 \\
0.0001 \\
0.0001 \\
\end{array}$ & $\begin{array}{l}0.9 \\
0.9 \\
0.9 \\
0.9 \\
\end{array}$ \\
\hline $\mathrm{C}_{4}$ & $8 b^{c}$ & $\begin{array}{l}0.69276 \\
0.19276 \\
0.69276 \\
0.19276\end{array}$ & $\begin{array}{l}0.98680 \\
0.01319 \\
0.48680 \\
0.51319\end{array}$ & $\begin{array}{l}0.35336 \\
0.64663 \\
0.85336 \\
0.14663 \\
\end{array}$ & $\begin{array}{l}0.0001 \\
0.0001 \\
0.0001 \\
0.0001\end{array}$ & $\begin{array}{l}0.9 \\
0.9 \\
0.9 \\
0.9\end{array}$ \\
\hline$C_{5}$ & $8 b^{c}$ & $\begin{array}{l}0.56318 \\
0.06318 \\
0.56318 \\
0.06318\end{array}$ & $\begin{array}{l}0.07783 \\
0.92216 \\
0.57783 \\
0.42216 \\
\end{array}$ & $\begin{array}{l}0.33428 \\
0.66571 \\
0.83428 \\
0.16571 \\
\end{array}$ & $\begin{array}{l}0.0001 \\
0.0001 \\
0.0001 \\
0.0001 \\
\end{array}$ & $\begin{array}{l}0.9 \\
0.9 \\
0.9 \\
0.9 \\
\end{array}$ \\
\hline $\mathrm{C}_{6}$ & $8 b^{c}$ & $\begin{array}{l}0.47538 \\
0.97538 \\
0.47538 \\
0.97538 \\
\end{array}$ & $\begin{array}{l}0.86254 \\
0.13745 \\
0.36254 \\
0.63745 \\
\end{array}$ & $\begin{array}{l}0.32270 \\
0.67730 \\
0.82270 \\
0.17730 \\
\end{array}$ & $\begin{array}{l}0.0001 \\
0.0001 \\
0.0001 \\
0.0001 \\
\end{array}$ & $\begin{array}{l}0.9 \\
0.9 \\
0.9 \\
0.9 \\
\end{array}$ \\
\hline$C_{7}$ & $8 b^{c}$ & $\begin{array}{l}0.34558 \\
0.84558 \\
0.34558 \\
0.84558\end{array}$ & $\begin{array}{l}0.95565 \\
0.04434 \\
0.45565 \\
0.54434\end{array}$ & $\begin{array}{l}0.30720 \\
0.69279 \\
0.80720 \\
0.19279\end{array}$ & $\begin{array}{l}0.0001 \\
0.0001 \\
0.0001 \\
0.0001\end{array}$ & $\begin{array}{l}0.9 \\
0.9 \\
0.9 \\
0.9\end{array}$ \\
\hline $\mathrm{C}_{8}$ & $8 b^{c}$ & $\begin{array}{l}0.25837 \\
0.75837 \\
0.25837 \\
0.75837\end{array}$ & $\begin{array}{l}0.74010 \\
0.25989 \\
0.24010 \\
0.75989 \\
\end{array}$ & $\begin{array}{l}0.28977 \\
0.71022 \\
0.78977 \\
0.21022 \\
\end{array}$ & $\begin{array}{l}0.0001 \\
0.0001 \\
0.0001 \\
0.0001\end{array}$ & $\begin{array}{l}0.9 \\
0.9 \\
0.9 \\
0.9 \\
\end{array}$ \\
\hline$C_{9}$ & $8 b^{c}$ & $\begin{array}{l}0.12798 \\
0.62798 \\
0.12798 \\
0.62798 \\
\end{array}$ & $\begin{array}{l}0.83215 \\
0.16784 \\
0.33215 \\
0.66784 \\
\end{array}$ & $\begin{array}{l}0.27067 \\
0.72932 \\
0.77067 \\
0.22932 \\
\end{array}$ & $\begin{array}{l}0.0001 \\
0.0001 \\
0.0001 \\
0.0001 \\
\end{array}$ & $\begin{array}{l}0.9 \\
0.9 \\
0.9 \\
0.9 \\
\end{array}$ \\
\hline$C_{10}$ & $8 b^{c}$ & $\begin{array}{l}0.04002 \\
0.54002 \\
0.04002 \\
0.54002\end{array}$ & $\begin{array}{l}0.61469 \\
0.38530 \\
0.11469 \\
0.88530\end{array}$ & $\begin{array}{l}0.25800 \\
0.74199 \\
0.75800 \\
0.24199\end{array}$ & $\begin{array}{l}0.0001 \\
0.0001 \\
0.0001 \\
0.0001\end{array}$ & $\begin{array}{l}0.9 \\
0.9 \\
0.9 \\
0.9\end{array}$ \\
\hline
\end{tabular}

Main interatomic distances (Å)

\begin{tabular}{|ll|ll|ll|}
\hline Sr/Bi-O & $2.3422 * 2$ & Ta-O & 1.9245 & P-O & 1.4423 \\
& 2.5011 & & 1.9410 & & 1.6371 \\
& $2.6335 * 2$ & & 1.9543 & & 1.6373 \\
& 2.7572 & & 2.0186 & P-C & 1.8594 \\
& 2.8135 & & 2.0941 & & \\
$2.8384 * 2$ & & 2.1478 & & \\
$2.9155 * 2$ & & & & \\
\hline 3.0539 & & & & \\
\hline
\end{tabular}

${ }^{a} A D P$ Isotropic atomic displacement parameter

${ }^{\mathrm{b}} \mathrm{SOF}$ site occupancy factor

${ }^{\mathrm{C}} \mathrm{Half}$ of the positions are occupied to avoid unrealistically close interatomic-intermolecular distances between $\mathrm{C}_{10} \mathrm{PO}_{3}$ chains and water molecules. 


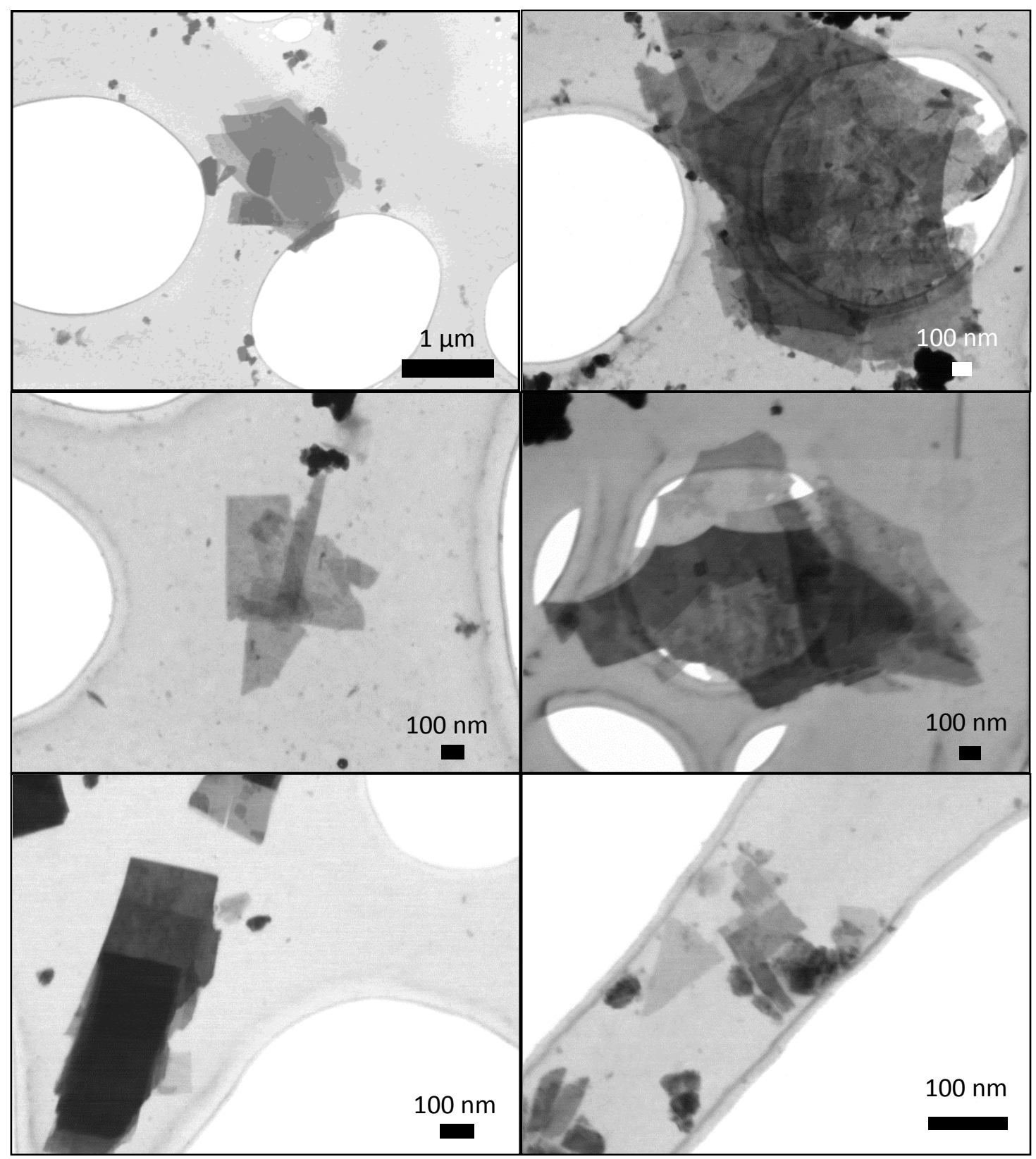

Figure S4. STEM/SEM images of nanosheets of exfoliated $\mathrm{C}_{10} \mathrm{PO}_{3}-\mathrm{HST}$.

Table S3. Inter-reticular distances of $\mathrm{C}_{10} \mathrm{PO}_{3}$-HST bulk (obtained from PXRD) and as nanosheets (obtained from FFT of HAADF STEM image). These distances are in accordance with the ones expected for perovskite structures. [3]

\begin{tabular}{|l|l|l|}
\hline & $\mathrm{d}_{100}(\mathrm{~nm})$ & $\mathrm{d}_{110}(\mathrm{~nm})$ \\
\hline PXRD & 0.3904 & 0.2759 \\
\hline FFT & 0.3987 & 0.2832 \\
\hline
\end{tabular}



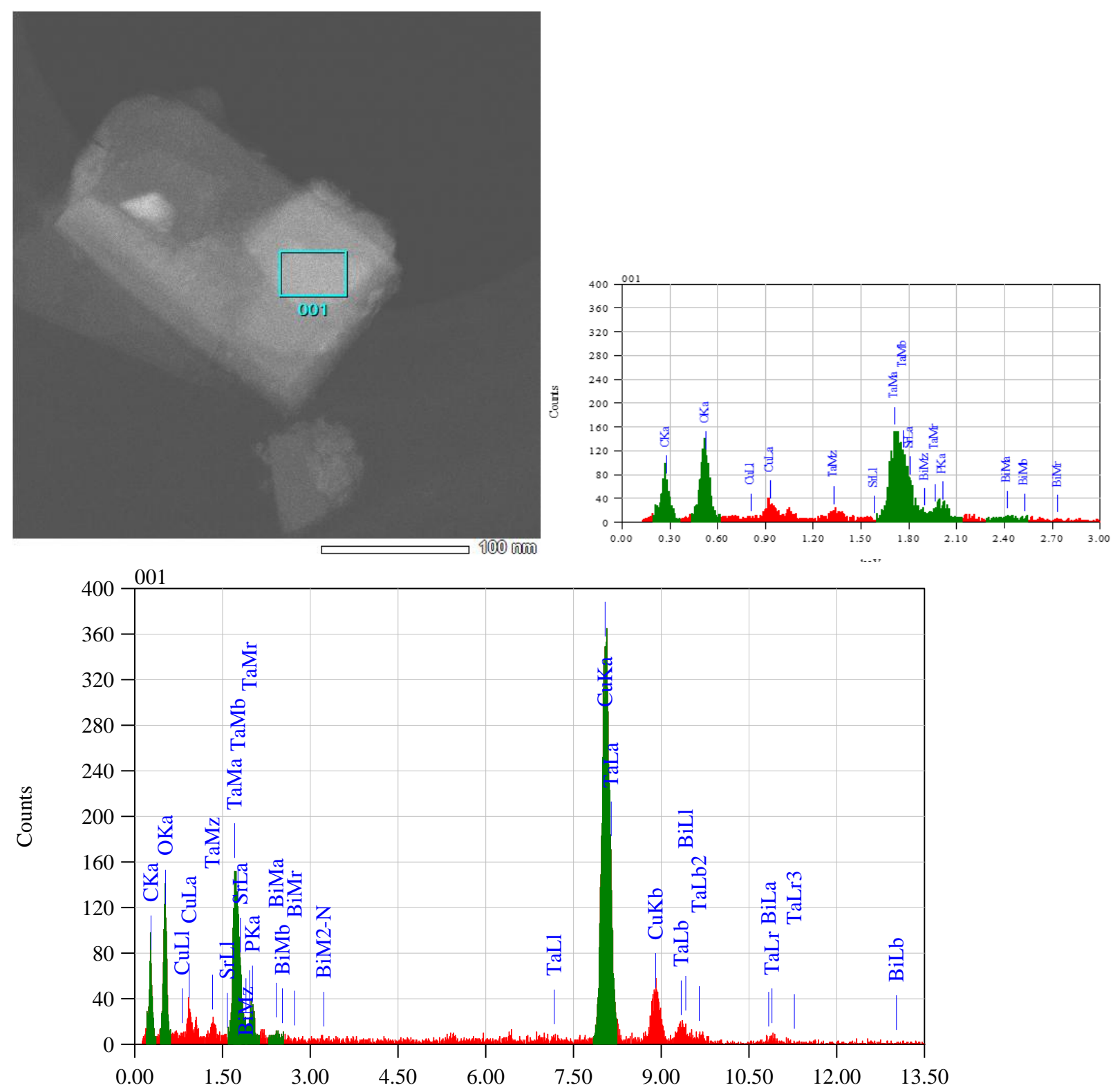

$\mathrm{keV}$

\begin{tabular}{|c|c|c|c|c|c|}
\hline Element & Energy (keV) & Mass (\%) & Counts & Sigma & Atom (\%) \\
\hline $\mathrm{CK}^{*}$ & \multicolumn{5}{|l|}{ Excluded } \\
\hline $\mathrm{OK}^{*}$ & \multicolumn{5}{|l|}{ Excluded } \\
\hline $\mathrm{PK}$ & 2.013 & 5.44 & 260.07 & 0.53 & 21.67 \\
\hline $\mathrm{CuK}^{*}$ & \multicolumn{5}{|l|}{ Excluded } \\
\hline $\mathrm{Sr} \mathrm{L}^{*}$ & 1.806 & 19.35 & 261.07 & 3.94 & 27.27 \\
\hline $\mathrm{Ta} \mathrm{M}^{*}$ & 1.709 & 72.33 & 1169.18 & 3.35 & 49.36 \\
\hline $\mathrm{Bi} \mathrm{L}^{*}$ & 2.419 & 2.88 & 31.30 & 1.23 & 1.70 \\
\hline
\end{tabular}

Figure S5. Example of TEM-EDX analysis on exfoliated $\mathbf{C}_{10} \mathbf{P O}_{3}-\mathbf{H S T}$. Green peaks in the EDX spectrum are the ones used for quantification. 

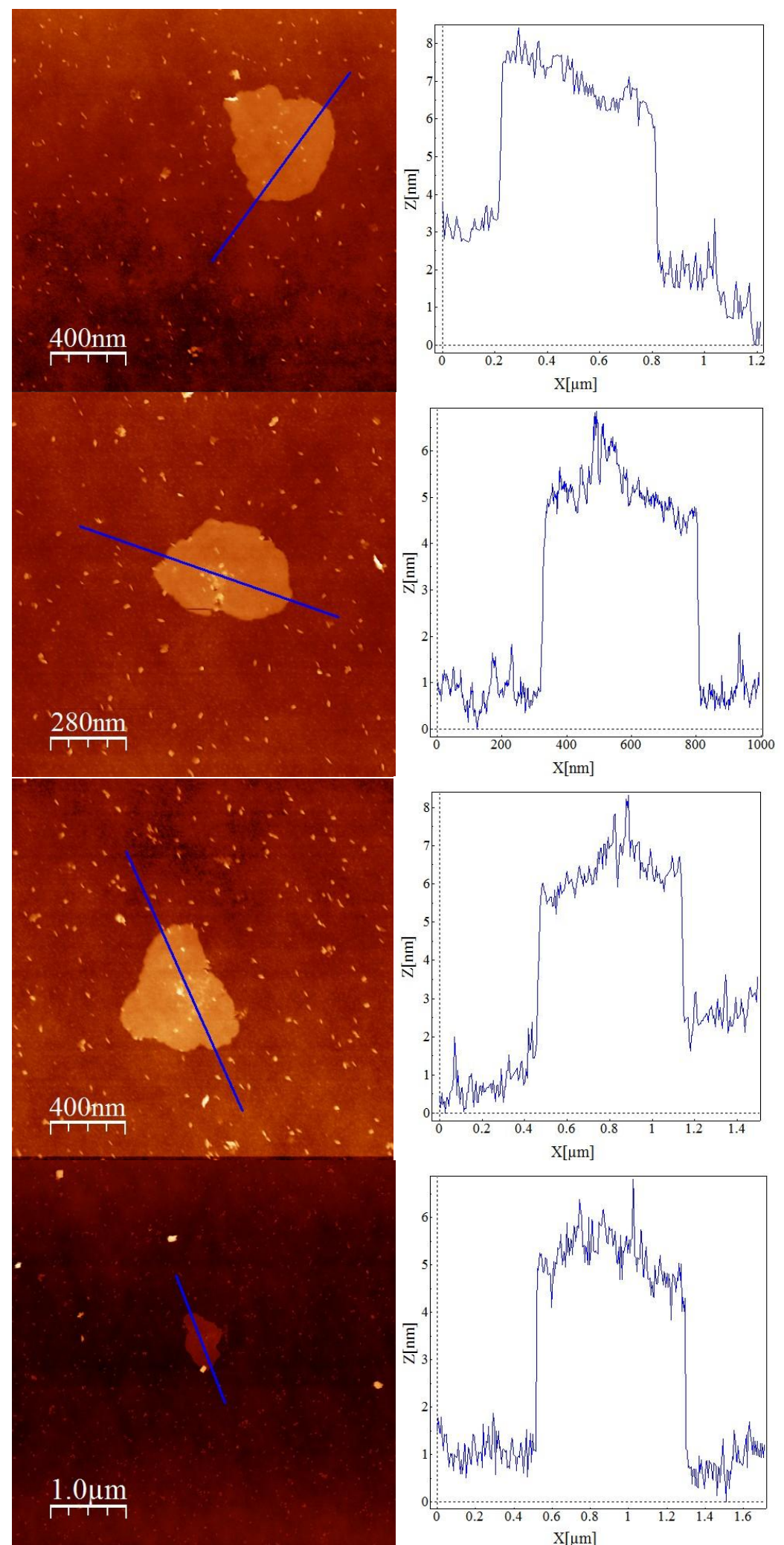

Figure S6. AFM images of $\mathrm{C}_{10} \mathrm{PO}_{3}-\mathrm{HST}$ nanosheets with the corresponding topographic profiles. 

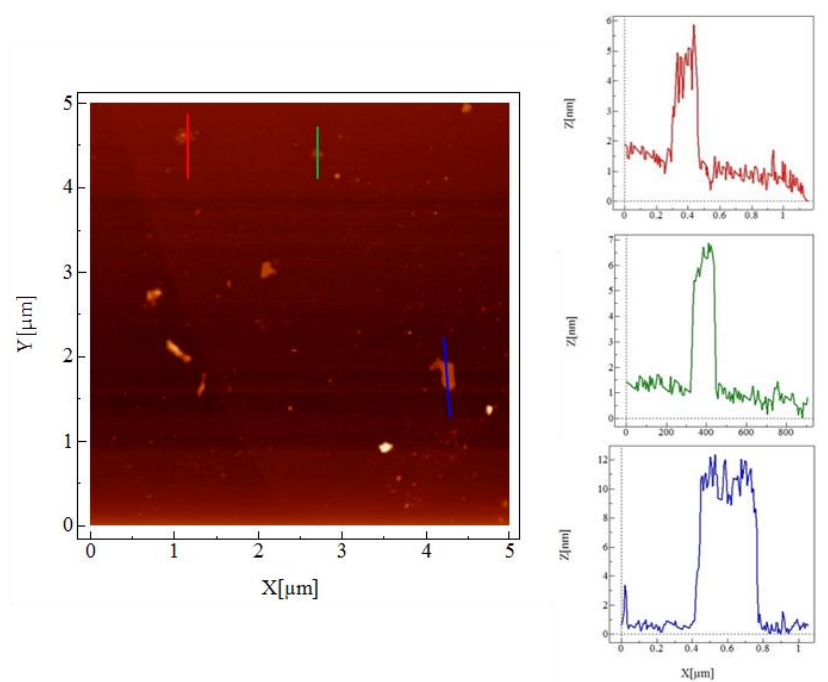

Figure S7. AFM image of a deposit of $\mathrm{C}_{10} \mathrm{PO}_{3}-\mathrm{HST}$ nanosheets with the corresponding topographic profiles.

\section{Light Scattering Analysis}

\section{Static light scattering}

The intensity scattered from a dilute colloidal suspension without long range interactions can be simply written as:

(equation S1)

$$
I(q) \propto \int n(V) V^{2}\left(1-\frac{q^{2} R_{g}^{2}(V)}{3}+O\left(q^{4}\right)\right) d V
$$

where $q$ is the scattering vector, $P(V)$ the number of colloids with volume $V$ and radius of gyration $R_{g}(V)$. The series expansion in this equation is valid in the Guinier regime $\left(q R_{g}<<1\right)$. Equation $S 1$ is very general and the radius of gyration of an object with a defined geometry can usually be expressed analytically in terms of its characteristic dimensions. Thus the radius of gyration of thin discs with diameter $d$ and thickness I is given by $\mathrm{R}_{\mathrm{g}}{ }^{2}=\mathrm{d}^{2} / 8+\mathrm{I}^{2} / 12$. In the so-called Zimm representation, i.e. $\mathrm{I}$ ${ }^{1}(q)$ as a function of $q^{2}$, the partial compensation of the different contributions to the $q^{4}$ term can allow one to estimate the radius of gyration outside of the Guinier regime by using a parabolic fit of the data instead of the conventional linear fit. [4]

The suspensions studied in the present manuscript are slowly sedimenting and this can complicate the extrapolations to $\mathrm{q}$ $=0$. Therefore, we first build interpolating functions through the experimental points measured at a given angle to compute interpolated values at a given same times for all scattering angles. The choice of the interpolation method affects a lot the errors bars on the extrapolated values. The data in Figure 11 were interpolated by fitting with an exponential function on the whole interval of time and this gave a very smooth $\mathrm{I}(\mathrm{q}=0)$. For comparison, Figure $\mathrm{S} 8$ shows the time evolution of the extrapolated $\mathrm{I}(\mathrm{q}=0)$ obtained after an interpolation by a sliding parabolic fit on seven data points centered on the given time, together with the one reported in Fig. 11.

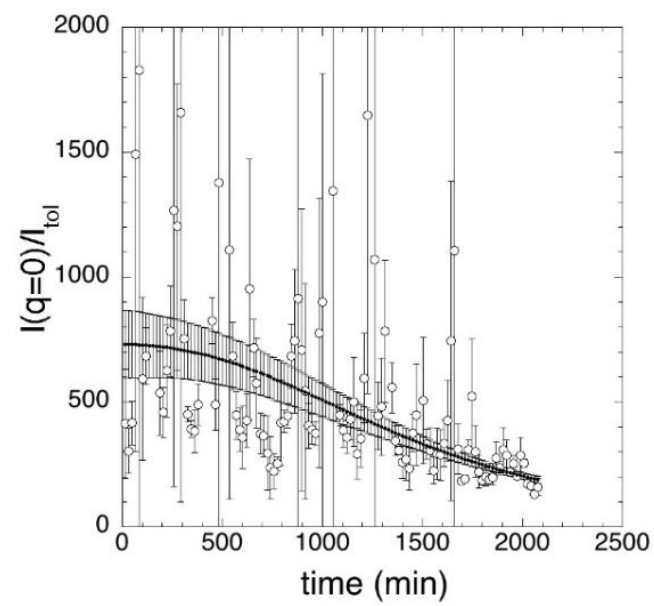

Figure S8. Time evolution of $\mathrm{I}(\mathrm{q}=0)$ : continuous line, values obtained from interpolating exponential fits; open symbols, values obtained with a sliding parabolic fit over seven data points. 
The estimated average radius of gyration of the nanosheets are very large and are measured well out of the Guinier regime. Therefore, we used parabolic fits over the five smaller scattering angles for the extrapolation in Zimm representation. A typical example is shown in Figure S9 The corresponding $\mathrm{qR}_{\mathrm{g}}$ values range between 2.8 and 7 at the beginning of the experiment and between 1.9 and 4.6 after 35 hours.

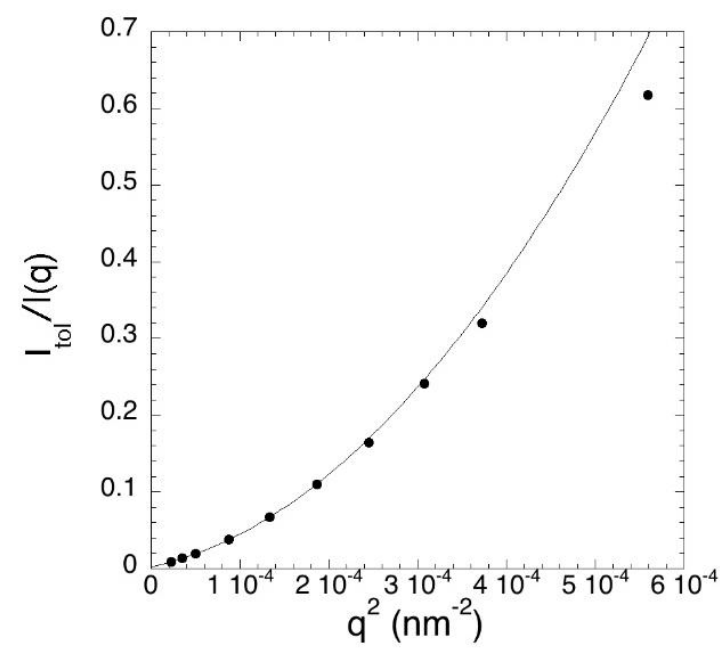

Figure S9. Typical Zimm representation of interpolated scattering intensity values. The continuous line is a parabolic fit over the five smallest q values. Interpolated values were obtained from the exponential interpolating fit.

\section{Dynamic light scattering}

The autocorrelation function of the scattered field is given by:

(equation S2)

$$
G^{(1)}(q, t)=\int n(V) V^{2}\left(1-\frac{q^{2} R_{g}^{2}(V)}{3}+O\left(q^{4}\right)\right) \exp \left(-q^{2} D(V) t\right) d V
$$

where $D(V)$ is the diffusion coefficient of colloids with volume $V$. For hard spheres, $D(V)$ is simply related to their radius $R$, $D(V)=D(R)=k_{B} T /(6 \pi \eta R)$. The same equation defines the equivalent Stokes-Einstein sphere radius $R_{h}$ corresponding to a value $D(V)$ for a non-spherical colloid. Depending on its shape, it can be related more or less easily to the dimensions of the colloid. For a thin disc with a small aspect ratio $(I<d) d=\pi R_{h} \cdot[5]$ Equation S2 defines an apparent size distribution $P(V, q)$ which can be only recovered by sophisticated methods. Indeed, a direct inverse Laplace transform of Eq. S2 is very sensitive to experimental noise. Moreover, a least squares fit of a discretized version of Eq. S2 is not possible since it corresponds to an ill-conditioned problem. Here we use the CONTIN software, a least squares fit approach with an added Tikhonov regularization taking into account $a$ priori conditions on the physically acceptable solutions.[6,7] For very polydisperse system where the conditions of the Guinier regime are not met for all colloids at each scattering angle, Eq. S2 can introduce an effective dependence of the measured diffusion coefficient on the scattering vector. In such a case, an estimation of the true constant diffusion coefficient can be obtained by extrapolating $D(q)$ as a function of $q^{2}$ to $q=0$.

Figure S10 left shows a typical correlation function together with the distribution of relaxation times obtained through CONTIN analysis while Figure S10 right shows the whole set of distribution of relaxation times as a function of time.
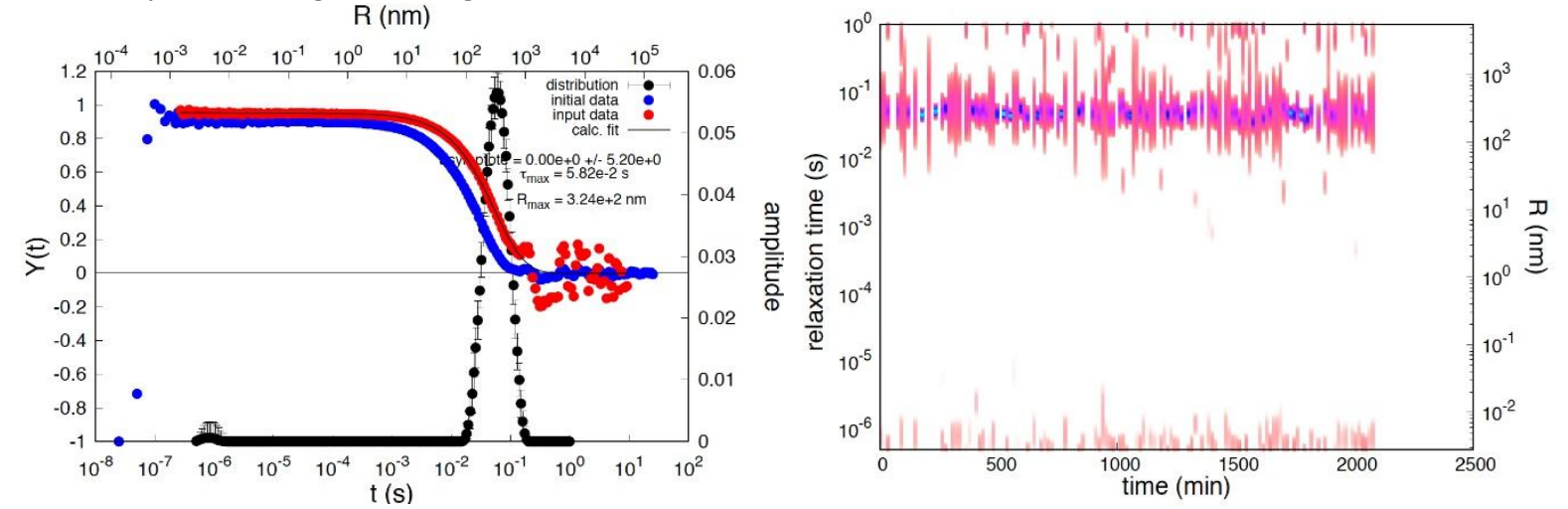

Figure S10. Left) Typical correlation functions together with the distribution of relaxation times obtained through CONTIN analysis: (blue) intensity correlation function minus 1; (red) electric field correlation function; (black) distribution of relaxation times; (continuous line) result of the CONTIN fit. Right) Set of distribution of relaxation times measured at scattering angle $30^{\circ}$ as a function of time. The color codes the amplitude of the distribution of relaxation times, increasing 
from pale pink to cyan. The top $x$-axis (left) and the right $y$-axis (right) give the equivalent Stokes-Einstein sphere radius corresponding to the relaxation time scale. CONTIN analysis gives consistently one single mode of relaxation with some spurious small peaks corresponding to unphysical hydrodynamic sizes.

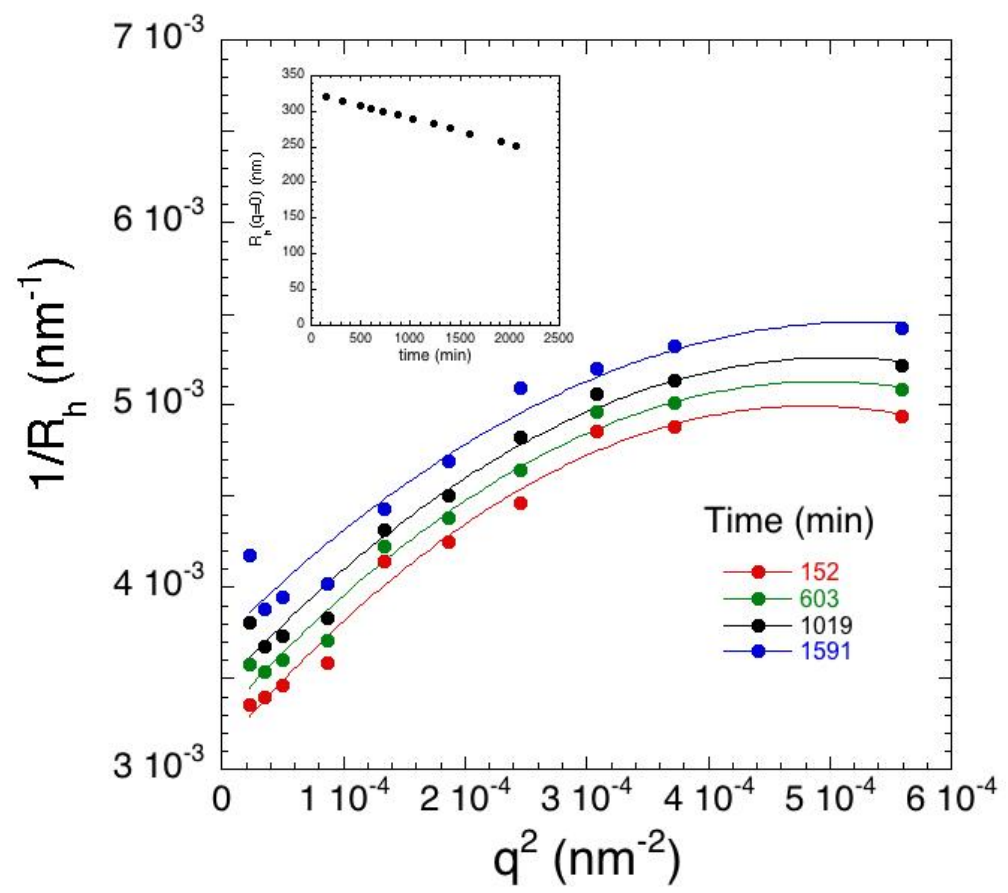

Figure S11. Typical plots of $1 / R_{h}(q)$ as a function of $q^{2}$. The inset shows the temporal evolution of $R_{h}(q=0)$ extrapolated with a parabolic fit of the data.

Figure S11 shows some representative parabolic fits of the variation of $1 / R_{h}(q)$ as a function of $q^{2}$ and the resulting $R_{h}(q=0)$ as a function of time. Due to the dispersion of data points, we choose to use a parabolic fit on the whole $q$ range instead of using the five smallest scattering angles as for the fits of the intensity in S9. In fact, whatever the choice of fitting method of these data, it would reflect the steady upward shift of the data with time, which translates in a steady decrease of the extrapolated hydrodynamic radius.

\section{References}

[1] K.R. Whittle, N.C. Hyatt, I.M. Reaney, Phase Transitions in Lanthanum-Doped Strontium Bismuth Tantalate, Chem. Mater. 20 (2008) 6427-6433. https://doi.org/10.1021/cm800475z.

[2] P. Juhás, T. Davis, C.L. Farrow, S.J.L. Billinge, PDFgetX3: a rapid and highly automatable program for processing powder diffraction data into total scattering pair distribution functions, J. Appl. Crystallogr. 46 (2013) 560-566. https://doi.org/10.1107/S0021889813005190.

[3] Y. Tsunoda, M. Shirata, W. Sugimoto, Z. Liu, O. Terasaki, K. Kuroda, Y. Sugahara, Preparation and HREM Characterization of a Protonated Form of a Layered Perovskite Tantalate from an Aurivillius Phase Bi2SrTa2O9 via Acid Treatment, Inorg. Chem. 40 (2001) 5768-5771. https://doi.org/10.1021/ic010266m.

[4] F. Schosseler, L. Leibler, An experimental study of gelation of chains, J. Phys. Lett. 45 (1984) $501-507$. https://doi.org/10.1051/jphyslet:019840045010050100.

[5] F.M. van der Kooij, A.P. Philipse, J.K.G. Dhont, Sedimentation and Diffusion in Suspensions of Sterically Stabilized Colloidal Platelets, Langmuir. 16 (2000) 5317-5323. https://doi.org/10.1021/la991571b.

[6] S.W. Provencher, A constrained regularization method for inverting data represented by linear algebraic or integral equations, Comput. Phys. Commun. 27 (1982) 213-227. https://doi.org/10.1016/0010-4655(82)90173-4.

[7] S.W. Provencher, CONTIN: A general purpose constrained regularization program for inverting noisy linear algebraic and integral equations, Comput. Phys. Commun. 27 (1982) 229-242. https://doi.org/10.1016/0010-4655(82)90174-6. 